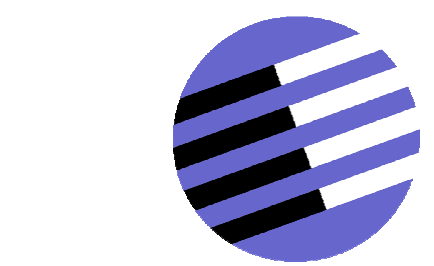

GOVERNANCE AND THE EFFICIENCY

OF ECONOMIC SYSTEMS

G ES Y

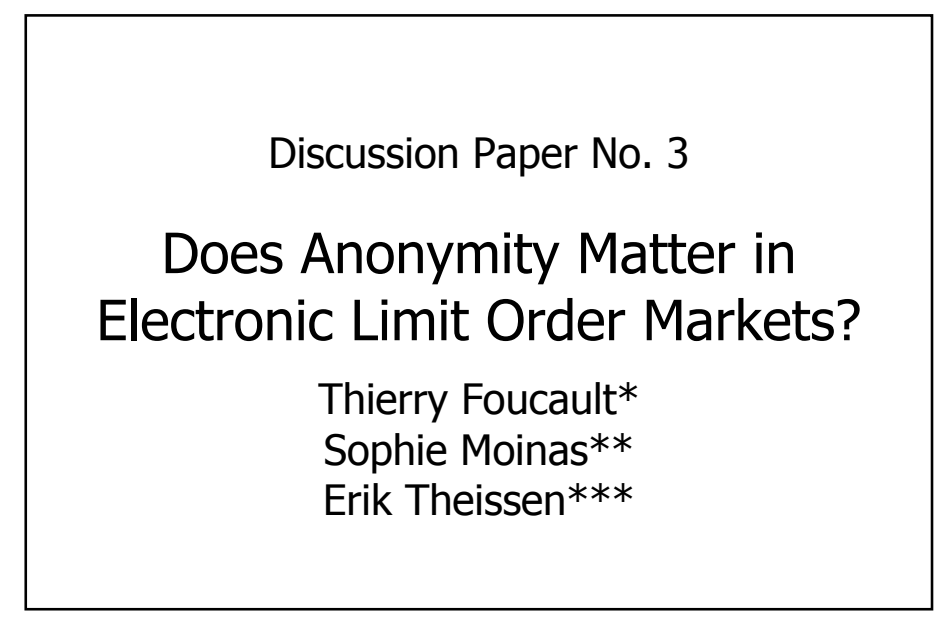

May 2004

*Thierry Foucault, HEC, School of Management, 78351, Jouy en Josas, France, Tel. (00)(33)139679411 **Sophie Moinas, HEC

$* * *$ Erik Theissen, Bonn University, Germany

Financial support from the Deutsche Forschungsgemeinschaft through SFB/TR 15 is gratefully acknowledged. 


\title{
DOES ANONYMITY MATTER IN ELECTRONIC LIMIT ORDER MARKETS? ${ }^{1}$
}

\author{
Thierry Foucault ${ }^{2}$ \\ HEC, School of Management, Paris (GREGHEC and CEPR) \\ and \\ Sophie Moinas \\ Doctorat HEC \\ and \\ Erik Theissen \\ Bonn University
}

This Draft: May, 2004

\footnotetext{
${ }^{1}$ We are grateful to J.Angel, B.Biais, P.Bossaerts, D. Brown, C. Caglio, F. Declerk, G.Demange, D.Leschinski, S. Lovo, R. Lyons, F.deJong, F.Palomino, C.Spatt, B. Rindi, R.Roll, G. Saar, D. Seppi and A.Whol for providing useful comments. We also received helpful comments from participants in various conferences (EFA03, WFA03, AFFI, INSEAD market microstructure workshop, the 6th ESC Toulouse-IDEI Finance Workshop) and seminars (Bielefeld University, CORE, Frankfurt University, Duisburg University, HEC Montreal, University of Amsterdam, University of Rotterdam, Tilburg University and Séminaire Bachelier). We thank Euronext Paris for providing the data. Financial support from the Fondation HEC is gratefully acknowledged. Of course, all errors or omissions are ours.

${ }^{2}$ Corresponding author. Thierry Foucault, HEC School of Management, 78351, Jouy en Josas, France.
} Tel: (00) (33) 1396794 11; Fax: (00) (33) 1396770 85; 


\begin{abstract}
"Does Anonymity Matter in Electronic Limit Order Markets?"

We develop a model of limit order trading in which some traders have better information on future price volatility. As limit orders have option-like features, this information is valuable for limit order traders. We solve for informed and uninformed limit order traders' bidding strategies in equilibrium when limit order traders' IDs are concealed and when they are visible. In either design, a large (resp. small) spread signals that informed limit order traders expect volatility to be high (resp. low). However the quality of this signal and market liquidity are different in each market design. We test these predictions using a natural experiment. As of April 23, 2001, the limit order book for stocks listed on Euronext Paris became anonymous. For our sample stocks, we find that following this change, the average quoted and effective spreads declined significantly. Consistent with our model, we also find that the size of the spread is a predictor of future price volatility and that the strength of the association between the spread and volatility is weaker after the switch to anonymity.
\end{abstract}

Keywords: Market Microstructure, Limit Order Trading, Anonymity, Transparency, Liquidity, Volatility Forecasts.

JEL Classification: G10, G14, G24 


\section{Introduction}

In the last decade, the security industry has witnessed a proliferation of electronic trading systems. Several of these new trading venues (e.g. Island for equity markets, Reuters D2000-2 for the foreign exchange market or MTS in bond markets) are organized as limit order markets where traders can either post quotes (submit limit orders) or hit posted quotes (submit market orders). This development has spurred considerable interest in understanding the trading process in these markets. Although significant progress has been achieved, there are still many unresolved questions. ${ }^{1}$ In particular, the impact of market design (transparency, priority rules etc...) on market liquidity and the informational content of the limit order book is still not well understood for limit order markets.

A case in point is the amount of information provided on traders' identities. Some markets (e.g. the Hong Kong Stock Exchange or the ASX) disclose, for each limit order standing in the limit order book, the issuing broker's identification code. In other markets (e.g. Island, Euronext or the NYSE), these brokers' IDs are concealed. Does it matter? How is market liquidity affected by the disclosure of limit order traders' identities? Is the informational content of the limit order book affected by anonymity? These questions are important as the effects of disclosing information about traders' identities and the nature of information contained in limit order books are constantly debated by practitioners, regulators and researchers. Our objective is to shed light on these issues, both theoretically and empirically.

Our analysis builds upon the idea that the limit order book contains information on the magnitude or the likelihood of future price changes (i.e. future price volatility). This claim follows from the fact that limit orders have option-like features. A trader who submits a sell (resp.buy) limit order on a security offers, for free, a call (resp.put) option on this security with a strike price equal to the price of the limit order. These options are valuable because traders monitoring the market can exercise them when there is a shift in the value of the security, by "picking off" stale limit orders. In order to cover the losses incurred when their limit orders are picked off, liquidity suppliers charge a bid-ask spread (see Copeland and Galai (1983)). As volatility is an important determinant of option values, information on future price volatility is valuable for limit order traders. It helps them to control their exposure to the risk of being picked-off by adequatly pricing their limit orders. Hence this information should be reflected into the prices posted in the limit order book.

In our model, we assume that some liquidity providers ("expert traders") have superior information on future price volatility. Specifically, expert traders have information on the likelihood

\footnotetext{
${ }^{1}$ Bloomfield, O'Hara and Saar (2003), Section 2, provide an excellent overview of the theoretical literature on limit order markets.
} 
of future price movements, which determines the risk of being picked off. Cautious bidding by expert traders, manifested by a large quoted spread, signals that this risk is large. We explore in details the implications of this remark. We show that a large spread can deter non-expert traders from improving upon the offers posted in the book. In turn, this effect induces expert traders to use "bluffing strategies". They sometimes try to "fool" non-expert traders by bidding as if the risk of being picked off were large (they post non-aggressive limit orders) when indeed it is small. When their bluff is successful, i.e. deters non-experts from improving upon posted quotes, experts earn larger profits. ${ }^{2}$

We compare the equilibrium outcome when the market is non-anonymous (limit order traders' IDs are visible) and the market is anonymous (limit order traders' IDs are concealed). A large quoted spread foreshadows a price movement and signals that the risk of being picked-off is large in either design. However, in the anonymous environment, uninformed traders cannot distinguish informative orders from non-informative orders. Accordingly, their bidding behavior is driven by their belief about the identity of the traders with orders in the limit order book. If expert traders represent a small fraction of the population submitting limit orders then a large spread is a weak signal that a price movement is pending. In this case, uninformed dealers are more likely to improve upon posted quotes in the anonymous environment. In contrast, if expert traders represent a significant fraction of the trading population then a large spread is a strong signal that a price movement is pending. In this case, uninformed traders are less likely to improve upon posted quotes in the anonymous environment. As for expert traders, they always bid more aggressively (i.e. bluff less frequently) when their identities are concealed than when they are not. Intuitively, their attempt to manipulate uninformed traders' beliefs is less effective in the anonymous environment. ${ }^{3}$

Ultimately, these effects determine the impact of a switch to anonymity on market liquidity and on the informativeness of the book. If the fraction of expert traders is small then a switch to anonymity makes all types of limit order traders more aggressive. Hence this switch reduces (i) the size of the quoted spread and (ii) the size of the effective spread (the difference between the execution price of a market order and the mid-quote). We also show that in this case a switch to anonymity reduces the informativeness of the size of the bid-ask spread for future price movements. Intuitively, the size of the spread is less informative because uninformed traders play

\footnotetext{
${ }^{2}$ In our model, a large quoted spread signals to potential competitors that the profitability of limit orders within the best quotes is small. This signal reduces potential competitors' incentive to enter more competitive orders in the book. This line of reasoning is reminiscent of Milgrom and Roberts (1982) or Harrington (1986) 's studies of limit pricing by a monopolist or oligopolists.

${ }^{3}$ Several market observers have pointed out that non-anonymity facilitates market manipulation. This problem has played an important role in the decision of the Tokyo Stock Exchange to switch to an anomymous trading system in July 2003. See "TSE witholds broker names in bid to deter speculators", Financial Times, July, 1 st, 2003.
} 
a more important role in determining bid-ask spreads.

We test these predictions using a natural experiment. This experiment takes opportunity of a change in the anonymity of the trading system owned by Euronext Paris (the French stock exchange). Euronext Paris operates an electronic limit order market where brokerage firms (henceforth broker-dealers) can place orders for their own account or on behalf of their clients. ${ }^{4}$ Until April 23, 2001 the identification codes for broker-dealers submitting limit orders were displayed to all brokerage firms. Since then, the limit order book is anonymous. Thus, using Euronext Paris data, we are able to empirically study the effect of concealing liquidity suppliers' identities and test some predictions of the model.

The empirical analysis supports our prediction that concealing liquidity suppliers' IDs affects the liquidity of a limit order market. Our experiment reveals a significant decrease in various measures of the quoted spread and the effective spread after the switch to an anonymous limit order book. These results are robust after controlling for changes in other variables which are known to affect bid-ask spreads (such as volatility and trading volume). We also find that the quoted depth (the number of shares offered at the best quotes), for various spread sizes, has increased following the switch to anonymity (although not significantly). Overall these findings suggest that the switch to anonymity has improved market liquidity.

Our empirical analysis also reveals that the limit order book contains information on the magnitude of future price changes. We divide each trading day into intervals of thirty minutes. We find that there is a positive and significant relationship between the magnitude of the price movement in one interval and the size of the spread in the previous interval. We also find that the strength of the association between price volatility and the lagged bid-ask spread is significantly smaller after the switch to anonymity. This finding is consistent with our model. Actually, in this model, a switch to anonymity reduces the informativeness of the bid-ask spread precisely when it improves market liquidity.

There is an intriguing contrast between our findings and the findings in the extant articles on the effects of anonymity in financial markets. ${ }^{5}$ These articles have primarily focused on the effects of providing information on the identities of the traders submitting marketable orders (liquidity demanders). Their common conclusion is that concealing information about liquidity demanders' identities impairs market liquidity. This conclusion rests on the fact that anonymity exacerbates adverse selection problems because it reduces liquidity suppliers' ability to screen informed and non-informed liquidity demanders. In contrast we focus on the effects of providing

\footnotetext{
${ }^{4}$ Many electronic limit order markets (e.g. the Toronto Stock Exchange, the Stockholm Stock Exchange or Island) have a design which is very similar to the trading system used by Euronext Paris.

${ }^{5}$ These include Seppi (1990), Forster and Georges (1992), Benveniste et al. (1992), Madhavan and Cheng (1997), Garfinkel and Nimalendran (2002), and Theissen (2003).
} 
information on the identities of the traders with limit orders in the book (liquidity suppliers). Our theoretical and empirical findings show that concealing information on liquidity suppliers' identities can improve market liquidity. These results underscore the complex nature of the issues related to anonymity in financial markets.

Finally our findings contribute to the recent literature on the informational content of the book (Irvine, Benston and Kandel (2000), Kalay and Whol (2002), Harris and Penchapagesan (2003), Cao, Hansch and Wang (2003)). This literature has analyzed whether book information (e.g. order imbalances) could be used to predict the direction of future price changes. In contrast, we study the informativeness of the book on future price volatility.

The remainder of the paper is organized as follows. Section 2 discusses the relevant literature. Section 3 describes a theoretical model of trading in a limit order market. In Section 4, we solve for equilibrium bidding strategies and we compare trading outcomes when liquidity suppliers' identities are disclosed and when they are concealed. Section 5 derives the empirical implications of our model and briefly discusses possible extensions. In Section 6, we empirically analyze the effect of concealing liquidity suppliers' identities using data from Euronext Paris. Section 7 concludes. The proofs which do not appear in the text are collected in the appendix. The notation used in the theoretical model is listed in Table 1 just before the Appendix.

\section{A Review of the Literature}

The provision of information on traders' identities improves market transparency. For this reason our paper is related to the longstanding controversy regarding the desirability of transparency in security markets (see O'Hara (1995) for a review). Recent papers have analyzed theoretically and empirically the effect of providing information on the prices and sizes of limit orders standing in the book (respectively Baruch (1999), Madhavan, Porter and Weaver (2002) and Boehmer, Saar and $\mathrm{Yu}(2003))$. However, none of these papers analyze the effect of disclosing information on limit order traders' identities, holding information on limit order sizes and prices constant. ${ }^{6}$

Waisburd (2003) analyzes empirically the effect of revealing traders' identities post-trade, using data from Euronext Paris. In contrast, we focus on the effect of revealing liquidity suppliers' IDs before a transaction. Waisburd (2003) considers a sample of stocks which trade under two different anonymity regimes: one in which the identities of the brokers involved in a transaction are revealed post trade and one in which they are concealed. He finds that the average bid-ask spread is larger and quoted depth is smaller in the post-trade anonymous regime. Interestingly,

\footnotetext{
${ }^{6}$ In Euronext Paris, intermediaries can observe all limit orders standing in the book (except hidden orders). This feature of the market has not been altered by the switch to anonymous trading.
} 
our empirical findings go in the opposite direction : the average bid-ask spread is smaller and the quoted depth is larger when liquidity suppliers' IDs are concealed. Hence post-trade and pre-trade anonymity have strikingly different effects.

Simaan, Weaver and Whitcomb (2003) argue that non-anonymous trading facilitates collusion among liquidity suppliers. Actually it is easier to detect and retribute dealers who breach a non-competitive pricing agreement when dealers' IDs are displayed. Simaan et al. (2003) find that dealers post more aggressive quotes in ECNs' than in Nasdaq, as predicted by the collusion hypothesis (dealers' IDs are displayed on Nasdaq but not in ECNs'). ${ }^{7}$ Our model does not rely on collusion among liquidity suppliers and thereby provides an alternative to Simaan et al. (2003)'s collusion hypothesis.

Rindi (2002) considers a rational expectations model (à la Grossman and Stiglitz (1980)). In the non-anonymous market, uninformed traders can make their offers contingent on the demand function of informed traders (their "limit orders") whereas they cannot in the anonymous market. With exogenous information acquisition, she shows that market liquidity is always smaller in the anonymous market. With endogenous information acquisition, she finds parameter values for which liquidity is higher in the anonymous market.

Our approach is distinct from Rindi (2002) because the nature of private information for liquidity suppliers is different. In our model, informed liquidity suppliers have information on the likelihood of a price movement but not on the direction of this price movement (more on this in Section 3.2). Furthermore the trading mechanism considered in this paper is different. Rindi (2002) analyzes a batch auction in which all orders are submitted simultaneously and are executed at a single clearing price. In contrast, in our model, liquidity suppliers submit their orders sequentially and, importantly, market orders can execute at different prices (they can "walk up" or "walk down" the book). This is closer to the actual operations of limit order markets.

For this reason, our paper is related to the recent literature on price formation in limit order markets (in particular Glosten (1994), Seppi (1997) and Sandås (2001)). Our baseline model can be seen as a (very) simplified version of Glosten (1994), with sequential bidding (as in Seppi (1997) or Sandås (2001)). In contrast to the extant literature however, we assume that some traders are better informed about the likelihood of a change in the asset value, i.e. the exposure of limit orders to "the risk of being picked-off". As these traders use this information to position their orders in the book, the state of the book provides information on future price volatility and

\footnotetext{
${ }^{7}$ Albanesi and Rindi (2000) also consider the effect of anonymity in a dealership market. The screen-based trading system used in the Italian bond market became anonymous in 1997. Albanesi and Rindi (2000) compare the time-series properties of transaction prices in this market before and after 1997. Due to data constraints, they cannot report results on direct measures of market liquidity such as quoted spread and depth, as we do in this paper.
} 
the risk of being picked off. This signaling role for the state of the book is new to this paper and is key for our results regarding anonymity.

\section{The Model}

\subsection{Timing and Market Structure}

We consider the following model of trading in a security market. There are 3 dates. At date 2 , the final value of the security, which is denoted $\widetilde{V}_{2}$, is realized. It is given by

$$
\widetilde{V}_{2}=v_{0}+\widetilde{\epsilon}_{1}
$$

where $\widetilde{\epsilon}_{1}$ is a random variable with zero mean. For simplicity we assume that $\widetilde{\epsilon}_{1}$ takes one of two values: $+\sigma$ or $-\sigma$ with equal probabilities. If an information event occurs at date 1 , a trader (henceforth a speculator) observes the innovation, $\epsilon_{1}$, with probability $\alpha .^{8}$ Upon becoming informed, the speculator can decide to trade or not. If, as happens with probability $(1-\alpha)$, no trader observes $\epsilon_{1}$ or if no information event occurs at date 1, a liquidity trader submits a buy or a sell market order with equal probabilities.

Each order must be expressed in terms of a minimum unit (a round lot) which is equal to $q$ shares. In the rest of the paper, we normalize the size of 1 round lot to 1 share $(q=1)$. The order size submitted by a liquidity trader is random and can be "small" (equal to 1 round lot) or "large" (equal to 2 round lots) with equal probabilities.

Following Easley and O'Hara (1992), we assume that there is uncertainty on the occurrence of an information event at date 1. Specifically, we assume that the probability of an information event is $\pi_{0}=0.5$. Figure 1 depicts the tree diagram of the trading process at date 1 . Liquidity suppliers (described below) post limit orders for the security at date 0. A sell (buy) limit order specifies a price and the maximum number of round lots a trader is willing to sell (buy) at this price. In the rest of this section we describe in more detail the decisions which are taken at dates 1 and 0 . Our modeling choices are discussed in detail in the next subsection.

Speculator. The speculator submits a buy or a sell order depending on the direction of his information. If $\epsilon_{1}$ is positive (negative), the speculator submits a buy (sell) market order so as

\footnotetext{
${ }^{8}$ An information event can be seen, for instance, as the arrival of public information (corporate announcements, price movements in related stocks, headlines news etc...). In this case, the probability $\alpha$ is the probability that a trader reacts to the new information before mispriced limit orders disappear from the book (either because a market order arrived or because limit order traders cancelled their orders); see Foucault, Roëll and Sandås (2003) for instance.
} 
to pick off all sell (buy) limit orders with a price below $v_{0}+\sigma$ (resp. above $\left.\left(v_{0}-\sigma\right)\right)$.

Liquidity Suppliers. Following Harris and Hasbrouck (1996), we assume that there are two kinds of liquidity suppliers: (a) risk-neutral value traders who post limit orders so as to maximize their expected profits and (b) pre-committed traders who have to buy or to sell a given number of round lots. Value traders can be viewed as brokers who trade for their own account. Precommitted traders represent brokers who seek to execute an order on behalf of a client (e.g. an institutional investor who rebalances his portfolio). ${ }^{9}$ Henceforth we will refer to the value traders as being "the dealers".

We assume that dealers are not equally informed on the likelihood of an information event. There are two types of dealers: (i) informed dealers who know whether or not an information event will take place at date 1 (but they do not know the direction of the event) and (ii) uninformed dealers who do not have this knowledge. Of course the risk of being picked off and thereby the cost of providing liquidity are larger when an information event is about to occur. For this reason, the schedule of limit orders posted by informed dealers is informative about the cost of liquidity provision.

Dealers post their limit orders sequentially, in 2 stages denoted $L$ (first stage) and $F$ (second stage). Figure 2 describes the timing of the bidding game which takes place at date 0 . With probability $(1-\beta)$, the price schedule (the limit order book) posted in the first stage is established by an informed dealer. Otherwise the limit order book is established by precommitted liquidity suppliers. In the second stage, an uninformed dealer observes the limit order book, updates her beliefs on the likelihood of an information event and decides to submit limit orders or not. This timing gives us the possibility to analyze how uninformed dealers react to the information contained in the limit order book. In the rest of the paper, we call the liquidity supplier acting in stage $L$ : the Leader and the liquidity supplier acting in stage $F$ : the Follower. Given this structure, $\beta$ should be interpreted as measuring informed dealers' "weight" in establishing the quotes.

At date 1, the incoming buy (sell) market order is filled against the sell (buy) limit orders posted in the book. Price priority is enforced and each limit order executes at its price. Furthermore, time priority is enforced. That is, at a given price, the limit order placed by the leader is executed before the limit order placed by the follower. Table 2 below lists the different types of traders in our model.

\footnotetext{
${ }^{9}$ Foucault, Kadan and Kandel (2003) show that it can be optimal for pre-committed traders to use limit orders instead of market orders.
} 
Table 2: The Traders

\begin{tabular}{|c|c|}
\hline Liquidity Suppliers (date 0) & Liquidity Demanders (date 1) \\
\hline Precommitted Limit Order Traders & Liquidity Traders \\
\hline Uninformed Dealers & Speculators \\
\hline Informed Dealers & \\
\hline
\end{tabular}

Limit Order Book. Modeling price formation in limit order markets quickly becomes very complicated. In order to keep the model tractable, we make the following assumptions. Liquidity suppliers can post sell limit orders at prices $A_{1}$ and $A_{2}$. We assume that

$$
A_{2}-A_{1}=A_{1}-v_{0}=\Delta>0 .
$$

The parameter $\Delta$ is the tick size, i.e. the minimum variation between two consecutive quotes in the book: $A_{1}$ is the smallest eligible price above the unconditional expected value of the asset and $A_{2}$ is the second smallest eligible price above this value. We describe the price schedule posted by liquidity supplier $j$ by the pair $\left(Q_{1 j}, Q_{2 j}\right)$ where $Q_{k j}$ denotes the number of round lots offered by liquidity supplier $j$ at price $A_{k}, k \in\{1,2\}$. We assume that $Q_{k j} \leq 2$. This assumption is innocuous because a market order submitted by a liquidity trader is at most for 2 round lots. It just simplifies the presentation of the results. We also assume that

$$
A_{1}<v_{0}+\sigma \leq A_{2}
$$

This assumption implies that limit orders posted at price $A_{1}$ are exposed to the risk of an information event but limit orders posted at price $A_{2}$ are not. Two implications follow. Collectively, dealers will never supply more than 2 round lots at price $A_{1}$ because this is the maximum demand of a liquidity trader. ${ }^{10}$ Furthermore dealers (informed or uninformed) can safely offer 2 round lots (the maximum size) at price $A_{2}$.

Thus, we can restrict our attention to the case in which the leader chooses one of 3 price schedules on the sell side: (a) $(0,2),(\mathrm{b})(1,2)$ and (c) $(2,2)$ that we denote $T, S$ and $D$, respectively. At the end of the first stage, the limit order book can be in one of 3 states: (a) "thin" if the leader posts schedule $T$, (b) "shallow" if the leader posts schedule $S$ or (c) "deep" if the leader posts schedule $D$. Given the state of the book, the uninformed dealer has three possible actions : (1) add 1 round lot at price $A_{1},(2)$ add 2 round lots at price $A_{1}$ or (3) do nothing. She never submits a limit order at price $A_{2}$ since this order has a zero execution probability (the leader always offers 2 round lots at price $A_{2}$ ). In summary, the follower chooses one of the following price

\footnotetext{
${ }^{10}$ Any round lot in excess of the 2 round lots executes only against orders submitted by the speculator because a liquidity trader never submits an order larger than 2 round lots.
} 
schedules: (a) $(1,0)$, (b) $(2,0)$ or (c) $(0,0)$. Each dealer chooses the schedule which maximizes his expected profit. The choice of pre-committed liquidity suppliers is exogenous: they choose schedule $K \in\{T, S, D\}$ with probability $0<\Phi_{K}<1$.

We make symmetric assumptions on the buy side. This symmetry implies that the equilibrium price schedules on the buy side are the mirror image of the equilibrium price schedules on the sell side. Thus from now on we focus on the sell limit orders chosen by the dealers exclusively. ${ }^{11}$

Consider the case in which a buy market order is submitted at date 1 and let $\widetilde{Q}\left(Q_{1}\right)$ be the size of this order when $Q_{1} \in\{0,1,2\}$ round lots are offered at price $A_{1}$ at date 1 . The buy market order can either be submitted by a liquidity trader or by a speculator. In the first case, the size of the market order is exogenous and can be for 1 or 2 round lots. We denote it by $\widetilde{Q}_{l} \in\{q, 2 q\}$. A speculator optimally chooses the size of his market order. We denote this size by $\widetilde{Q}_{s}$. If $\epsilon_{1}=\sigma$, a speculator optimally picks off all sell limit orders placed at price $A_{1}$ (since $A_{1}<v_{0}+\sigma$ ) which implies $\widetilde{Q}_{s}=Q_{1}$. We deduce that :

$$
\widetilde{Q}\left(Q_{1}\right)=I Q_{1}+(1-I) \widetilde{Q}_{l},
$$

where $I$ is an indicator variable equal to 1 if the trader submitting a buy market order at date 1 is informed and zero otherwise.

Anonymous and Non-Anonymous Limit Order Markets. We shall distinguish two different trading systems: (i) the anonymous limit order market and (ii) the non-anonymous limit order market. In the non-anonymous trading system, the follower observes the identity of the leader, that is she can distinguish between informative and non informative orders. In the anonymous market, she cannot. In both cases, however, the follower observes the price schedules posted in the first stage (i.e. the book is "open").

Measures of Market Liquidity We will compare the liquidity of these two trading systems for fixed values of the exogenous parameters $(\sigma, \alpha, \beta, \Delta)$. We compute two different measures of market liquidity: (a) the small trade spread (or quoted spread) which is the difference between the best ask price and the unconditional expected value of the security and (b) the large trade spread which is the difference between the marginal execution price of a market order for 2 round lots and the unconditional expected value of the security. For instance, if the first round lot executes at price $A_{1}$ and the second round lot executes at price $A_{2}$, the large trade spread is

\footnotetext{
${ }^{11}$ As we restrict bidders to 2 quotes on each side of the book, our model is best viewed as a model of competition at the inner quotes in the book. Several empirical papers (e.g. Biais, Hillion and Spatt (1995)) find that most of the activity is at, or close to, the best quotes.
} 
$\left(A_{2}-v_{0}\right)$. The large trade spread is a measure of price impact and is conceptually similar to the effective spread in our empirical analysis.

The expected small trade spread in a given trading mechanism is given by:

$$
\begin{aligned}
E S_{\text {small }} & =\operatorname{Pr}\left(Q_{1} \geq 1\right) A_{1}+\operatorname{Pr}\left(Q_{1}=0\right) A_{2}-v_{0} \\
& =\Delta\left(1+\operatorname{Pr}\left(Q_{1}=0\right)\right) .
\end{aligned}
$$

The expected large trade spread is given by

$$
E S_{l \text { arg } e}=\operatorname{Pr}\left(Q_{1}=2\right) A_{1}+\left(1-\operatorname{Pr}\left(Q_{1}=2\right)\right) A_{2}-v_{0},
$$

which rewrites

$$
E S_{l \arg e}=\Delta\left(2-\operatorname{Pr}\left(Q_{1}=2\right)\right) .
$$

Notice that the measures of market liquidity are determined by the probability distribution of the quoted depth $\left(Q_{1}\right)$ at the end of the bidding stage. As shown in Section 5, for some parameter values, a switch to anonymity reduces the small trade spread but simultaneously increases the large trade spread. Market liquidity unambiguously improves when both the small trade spread and the large trade spread decrease.

\subsection{Discussion.}

Informed Dealers. Declerk (2001) shows that there are substantial variations in the trading profits of the intermediaries who actively trade for their own account on Euronext Paris. This finding suggests that some intermediaries (those with superior profits on average) have more expertise, i.e. have an edge in positioning their quotes in the limit order book.

In our model, this expertise comes from superior information on the likelihood of a future price movement. Alternatively, we could have assumed that informed dealers have information on the magnitude of upcoming price changes (i.e. $\sigma$ ). The results in this case are identical to those we obtain. In both cases informed dealers have information on future price volatility $\left(\operatorname{Var}\left(\epsilon_{1}\right)\right)$. Information on future price volatility is useful for limit order traders because it helps them to correctly assess their exposure to the risk of being picked off and to position their quotes accordingly.

It is worth stressing that, in our model, informed dealers have information on future price volatility but not on the direction of future price movements. ${ }^{12}$ In particular, observe that the expected value of the security at date 0 is the same (and equal to $v_{0}$ ) for informed and uninformed

\footnotetext{
${ }^{12}$ As an example, consider the case of a dealer who knows that a merger announcement is pending. Numerous
} 
dealers alike. Hence it cannot be optimal for an informed dealer to trade against the book (since bid-ask prices are positioned around $v_{0}$ ). In other words, information on future price volatility is useful for limit order trading but useless for market order trading.

Some empirical findings suggest that some liquidity suppliers are able to correctly forecast the magnitude of future price movements. For instance, Lee, Mucklow and Ready (1993) find that the reduction in quoted depth and the increase in spread which precede earnings announcements are greater for announcements which trigger large price movements. They conclude (p.368) that: "Both findings suggest a market in which the liquidity suppliers are able to anticipate, to some extent, the price informativeness of an upcoming earnings release." Anand and Martell (2001) find that limit orders placed by institutional investors on the NYSE perform better than those placed by individuals, even after controlling for order characteristics (such as order aggressiveness or order size). They argue (p.2) that institutional investors are better able "to predict at least the flow of information and use this knowledge to submit trades, which avoid adverse selection associated with limit orders".

There is also anedoctal evidence that less informed traders actively use the information contained in limit orders. For instance, a recent consultation paper of the Australian Stock Exchange notes that (p.7) ${ }^{13}$ :
"Broker ids are an additional piece of information that can, in some circumstances, be useful in predicting future market activity. It is apparent that some traders attempt to second-guess future price movements based on trading by particular brokers [...] This activity has the ability to stifle and suppress natural liquidity, and imposes extra costs on participants when they try to disguise their trading strategies to protect their positions"

Also, on Euronext Paris, some intermediaries bitterly complained that it was more difficult for them to piggy-back on the orders placed by large (and presumably expert) intermediaries when the limit order book became anonymous. ${ }^{14}$

Timing. In our model, the informed dealer always submits his limit orders before the follower. A more general formulation would allow the sequence in which the informed and the uninformed empirical studies have shown that this type of announcement has no impact on the price of the acquiring firm, on average. Thus a dealer with this information can correctly anticipate that the announcement will trigger a price reaction for the acquiring firm without being able to predict the direction of the price reaction. Calcagno and Lovo (2001) or Rindi (2002) consider models in which liquidity suppliers possess directional information.

\footnotetext{
${ }^{13}$ See "ASX market reforms-Enhancing the liquidity of the Australian equity markets".

${ }^{14}$ See the following newspaper article : "L'anonymat gêne les professionnels", La Tribune, April 24th, page 1.
} 
dealer choose their price schedules to be random. ${ }^{15}$ This formulation however would obscure the presentation of our results without adding new insights. Actually, the follower's bidding strategy depends on the identity of the leader only when (i) the leader has a chance to be informed and (ii) the follower is uninformed. This configuration is therefore the only case in which concealing the leader's identity has an effect, if any.

Pre-committed Traders. Obviously, a switch to anonymity prevents traders from distinguishing informative and non-informative limit orders. Thus it blurs the inferences which can be drawn from the limit order book. In order to capture this effect, we have introduced precommited limit order traders in our model. By assumption, the orders placed by these traders contain no information. Hence, the larger is $\beta$, the smaller is the probability that the best quotes have been set by traders with information. In a sense, pre-committed traders play the role ascribed to noise traders in Noisy Rational Expectations models (e.g. Hellwig (1980)). As in many of these models, the behavior of these traders is taken as being exogenous.

\section{Equilibria in Anonymous and Non-Anonymous Limit Order Markets}

In this section, we analyze the nature of equilibria in the anonymous and in the non-anonymous market. We proceed as follows. First, as a building block, we study the follower's optimal reaction in each possible state of the book for given, but arbitrary, beliefs $\pi$ about the occurrence of an information event. Second, we study the benchmark case in which dealers have symmetric information (the leader and the follower are uninformed). Then we consider the case in which dealers have asymmetric information. In this case we first consider the regime in which the market is anonymous and eventually the non-anonymous regime.

\subsection{The Follower's Optimal Reaction}

Consider the case in which the follower observes a thin book $(K=T)$ at the end of the first stage. If she places a sell limit order for one round lot at price $A_{1}$ then her profit in case of execution

\footnotetext{
${ }^{15}$ In auctions with fixed end times, expert bidders may choose to place their bids in the closing seconds of the auction to avoid revealing their information (see the empirical study of Roth and Ockenfels (2002)). In limit order markets, the notion of fixed end time does not apply since the times at which market orders arrive are random. Thus an informed bidder who chooses to wait in order to avoid revealing his information runs the risk of missing the next trade. In addition, he cannot be certain that an uninformed bidder will not react before the arrival of the next market order. In these conditions, it is natural to assume that bidders' arrival times are random.
} 
is :

$$
A_{1}-V
$$

Consequently, her expected profit conditional on execution is

$$
A_{1}-E_{\pi}(V \mid \widetilde{Q}(1) \geq 1)
$$

where $\pi$ is the follower's belief on the occurence of an information event. In case of execution, the follower deduces that the size of the market order is at least equal to 1 round lot. This explains why the follower's valuation (conditional on execution) is given by an "upper-tail expectation" (see Glosten (1994)). Computations yield

$$
E_{\pi}(V \mid \widetilde{Q}(1) \geq 1)=v_{0}+\pi \alpha \sigma
$$

Now consider the case in which the follower offers another round-lot at price $A_{1}$ when one is already offered. Using the same reasoning, we deduce that the follower's expected profit on the second round lot is

$$
A_{1}-E_{\pi}(V \mid \widetilde{Q}(2) \geq 2) \text {. }
$$

Computations yield

$$
E_{\pi}(V \mid \widetilde{Q}(2) \geq 2)=v_{0}+\left(\frac{2 \pi}{\pi \alpha+1}\right) \alpha \sigma
$$

It is useful to interpret $E_{\pi}(V \mid \widetilde{Q}(1) \geq 1)$ as the "cost" of providing 1 round lot at price $A_{1}$ for a dealer who assigns a probability $\pi$ to the occurrence of an information event. Similarly $E_{\pi}(V \mid \widetilde{Q}(2) \geq 2)$ is the cost of providing one additional round lot at price $A_{1}$ when one is already offered. ${ }^{16}$ For this reason, we refer to the cost schedule defined by Equations (8) and (10) as being the expected cost of liquidity provision. ${ }^{17}$ This schedule is increasing (in the quantity) since

$$
\left(\frac{2 \pi}{\pi \alpha+1}\right) \alpha \sigma>\pi \alpha \sigma \quad \forall \pi>0 .
$$

The informed speculator always exhausts the depth available at price $A_{1}$. In contrast, a liquidity trader always trades at least 1 round lot but not necessarily 2 round lots. Thus the second round lot offered at price $A_{1}$ is relatively more exposed to the risk of being picked off than the first round lot. This explains why the cost of providing this second round lot is larger than the cost

\footnotetext{
${ }^{16}$ For a given $\pi$, the cost of providing a second round lot at price $A_{1}$ does not depend on whether the trader offering the second round lot is also the trader offering the first round lot or not. Actually if the two traders are different, the first one has time priority. Thus the first round lot will be executed before the second. Hence execution of the second round lot means that the market order size is larger than or equal to 2 round lots.

${ }^{17}$ The actual cost is either high if an information event occurs or low (and equal to zero here) if there is no information event.
} 
of providing the first one. Hence it may be optimal (depending on parameter values) to offer 1 round lot at price $A_{1}$, but not more.

When the state of the book is informative, the follower's belief about the occurence of an information event, $\pi$, will depend on the state of the book just before she submits (or not) her limit order. Henceforth, to make this linkage explicit, we denote by $\pi_{K}$ the follower's belief when the state of the book, at the end of stage $L$, is $K\left(\pi_{K}\right.$ is endogenized in section 4.3).

Equations (7) and (9) imply that the follower perceives the expected profit on the marginal round lot offered at price $A_{1}$ as being

$$
A_{1}-E_{\pi_{K}}\left(V \mid \widetilde{Q}\left(Q_{1}\right) \geq Q_{1}\right),
$$

where $Q_{1}$ is the total number of round lots offered at price $A_{1}$ at the end of the bidding stage. For a given state of the book at the end of stage $L$, the follower must optimally fill the book up to the point where an additional round lot offered at price $A_{1}$ would lose money (as first pointed out by Seppi (1997) and Sandas (2001)). This means that the follower fills the book in such a way that eventually $Q_{1}^{*}$ round lots are offered at price $A_{1}$ where $Q_{1}^{*}$ is the largest integer in $\{1,2\}$ such that

$$
A_{1}-E_{\pi_{K}}\left(V \mid \widetilde{Q}\left(Q_{1}^{*}\right) \geq Q_{1}^{*}\right) \geq 0 .
$$

If this inequality cannot be satisfied for $Q_{1}^{*} \in\{1,2\}$ then $Q_{1}^{*}=0$ (the book is empty at price $A_{1}$ ). Using this remark and Equations (8) and (10), the follower's optimal behavior for each possible state of the book is easily derived. It is given by the next lemma.

\section{Lemma 1 :}

1. When the follower observes a thin book, she submits a limit order at price $A_{1}$ for 2 round lots if $\frac{2 \pi_{T} \alpha \sigma}{\pi_{T} \alpha+1}<\Delta, 1$ round lot if $\pi_{T} \alpha \sigma<\Delta<\frac{2 \pi_{T} \alpha \sigma}{\pi_{T} \alpha+1}$ and does nothing otherwise.

2. When the follower observes a shallow book, she submits a limit order at price $A_{1}$ for 1 round lot if $\frac{2 \pi_{S} \alpha \sigma}{\pi_{S} \alpha+1}<\Delta$ and does nothing otherwise.

3. When the follower observes a deep book, she does nothing.

The risk of being picked off is large when the likelihood of an information event is large. For this reason the expected cost of liquidity provision increases with the likelihood of an information event (see Equations (8) and (10)). Hence the follower's inclination to add depth to the book is smaller when she assigns a large probability to the occurrence of an information event. This effect explains why, for a given state of the book, the follower acts less and less aggressively as the likelihood of an information event, $\pi_{K}$, increases. 


\subsection{A Benchmark : Symmetric information.}

When dealers have symmetric information on future price volatility, the state of the book at the end of the first stage does not convey information on the actual cost of liquidity provision to the follower. For this reason, the follower's beliefs about this cost are unaffected by the state of the book and the level of information on traders' IDs. Therefore $\pi_{S}=\pi_{T}=\pi_{0} \stackrel{\text { def }}{=} 0.5$ in both the anonymous and the non-anonymous trading systems.

In this case, it follows from the reasoning in the previous subsection that, in equilibrium, the number of round lots offered at price $A_{1}$ at the end of the bidding stage is the largest $Q_{1}^{*}$ in $\{1,2\}$ such that:

$$
A_{1}-E_{\pi_{0}}\left(V \mid \widetilde{Q}\left(Q_{1}^{*}\right) \geq Q_{1}^{*}\right) \geq 0
$$

and if this inequality cannot be satisfied for $Q_{1}^{*} \in\{1,2\}$ then $Q_{1}^{*}=0$. Observe that $Q_{1}^{*}$ in this case does not depend on the state of the book at the end of the first stage ( $K$ does not play a role in Inequality (13)). Also, and more importantly, $Q_{1}^{*}$ does not depend on whether or not the market is anonymous. It immediately follows that the liquidity of the limit order market is not affected by the provision of information on traders' IDs in this case.

Proposition 1 (Benchmark): When dealers have symmetric information, market liquidity (i.e. the small trade spread and the large trade spread) is identical in the anonymous and in the nonanonymous trading system.

This result will not hold when there is asymmetric information among dealers, as shown in Corollary 2 (Section 4.4). The exact value of $Q_{1}^{*}$ depends on the parameters. Using Equations (8) and (10), it is immediate that $Q_{1}^{*}=2$ iff

$$
\frac{2 \pi_{0} \alpha \sigma}{\pi_{0} \alpha+1}=\frac{2 \alpha \sigma}{\alpha+2}<\Delta
$$

The next proposition describes the equilibrium bidding strategies of each dealer in equilibrium when this condition is satisfied.

Proposition 2 (Benchmark): Suppose that dealers have symmetric information. When $\frac{2 \alpha \sigma}{\alpha+2}<$ $\Delta$, the unique subgame perfect equilibrium is as follows: (i) the dealer acting in stage $L$ chooses schedule $D$ and (ii) the follower acts as described in Lemma 1 for $\pi_{S}=\pi_{T}=0.5$. In equilibrium, the book obtained at the end of the second stage is always deep (2 round lots are offered at price $\left.A_{1}\right)$, i.e. the small trade spread and the large trade spread are equal to $A_{1}-v_{0}$. 
Observe that when she observes a large spread, the follower submits a limit order establishing the small spread. Anticipating this reaction, the dealer acting in stage $L$ offers 2 round lots at price $A_{1}$, leaving no possibility of entry to the follower.

In the rest of the paper, we will assume that the parameters satisfy Condition (14). This restriction on the parameters does not affect the findings regarding anonymity but it simplifies the presentation of the paper. Actually it limits the number of subcases that must be analyzed to describe the equilibrium. Furthermore, this restriction helps us to better focus the analysis on the driving force behind our results : a large spread can deter the follower from improving upon the best quotes because it signals an impending information event. This effect can be nonambiguously ascribed to asymmetric information if it does not arise otherwise, i.e. if the follower always improves upon a large spread when dealers have identical information. The condition on the parameters guarantees that this is the case as shown by the previous proposition.

\subsection{The Anonymous Limit Order Market}

Now we turn to the case in which there is asymmetric information among dealers. In this subsection we analyze equilibrium bidding strategies when the limit order market is anonymous. Throughout we focus on Perfect Bayesian equilibria of the bidding game at date 0, as usual in analyses of signaling games. We denote by $\Psi$ an indicator variable which is equal to 1 if there is an information event and zero otherwise. To make things interesting, we focus on the case in which:

$$
\frac{2 \alpha \sigma}{\alpha+2}<\Delta<\alpha \sigma
$$

The Left Hand Side of this inequality just restates Condition (14). The Right Hand Side implies that when there is an information event, limit orders placed at price $A_{1}$ do not yield positive expected profits. ${ }^{18}$ Actually, the actual cost of providing 1 round lot at price $A_{1}$ if there is an information event is (see Eq.(8)):

$$
E_{1}(V \mid \widetilde{Q}(1) \geq 1)=v_{0}+\alpha \sigma
$$

which is larger than $A_{1}=v_{0}+\Delta$ when $\Delta<\alpha \sigma$. The cost of providing 2 round lots is even larger since the cost of liquidity provision at price $A_{1}$ increases with the quantity supplied at this price.

Thus when the informed dealer knows that an information event is about to take place, he cannot profitably place a limit order at price $A_{1}$. For this reason we shall focus on equilibria in

\footnotetext{
${ }^{18}$ Clearly the set of parameters such that Condition (15) is satisfied is never empty. We have also assumed: $\sigma \leq 2 \Delta$. This constraint combined with the R.H.S of Condition (15) imposes $\alpha>\frac{1}{2}$. This condition can be relaxed if the condition $\sigma \leq 2 \Delta$ is relaxed. Intuitively, the risk of informed trading matters only if $\alpha$ or $\sigma$ are large enough.
} 
which the informed dealer posts a large spread (chooses schedule $T$ ) when there is an information event. When there is no information event, the informed dealer can profitably establish the deep book. He then obtains an expected profit equal to:

$$
\Pi^{L}(D, 0) \stackrel{\text { def }}{=}\left(A_{1}-v_{0}\right) E\left(\widetilde{Q}_{u}\right)=\frac{3\left(A_{1}-v_{0}\right)}{2}>0 .
$$

But he may also try to reap a larger profit by quoting a large spread (the less competitive schedule $T$ ). If the informed dealer sometimes behaves in this way, we say that he follows a bluffing strategy.

For the follower, a large spread constitutes a warning : maybe the spread is large because the leader knows that an information event is pending. Accordingly she revises upward the probability she assigns to an information event (see Eq.(17) below). If this revision is large enough, she is deterred from submitting a limit order within the best quotes and the informed dealer clears all the market orders at price $A_{2}>A_{1}$. His bluff has been successful.

Formally let $m$ be the probability with which the informed dealer chooses schedule $D$ when $\Psi=0$. With the complementary probability, he chooses schedule $T$ when $\Psi=0$. The next proposition describes the conditions under which there exists an equilibrium with bluffing (i.e. $0 \leq m<1)$. Let $\beta^{*} \stackrel{\text { def }}{=} \frac{(\alpha-r)}{(\alpha-r)+\Phi_{T}(2 r-\alpha)}$ and $r \stackrel{\text { def }}{=} \frac{\Delta}{\sigma}$.

Proposition 3 : When $0 \leq \beta \leq \beta^{*}$ and $\frac{2 \alpha \sigma}{\alpha+2}<\Delta<\alpha \sigma$, the following bidding strategies constitute an equilibrium:

1. When there is an information event, the informed dealer posts schedule $T$. When there is no information event, the informed dealer posts schedule $D$ with probability $m^{*}(\beta)=$ $\left(\frac{\left(1-\beta+\beta \Phi_{T}\right)}{1-\beta}\right)\left(\frac{2 r-\alpha}{r}\right)$ and schedule $T$ with probability $\left(1-m^{*}(\beta)\right)$, with $0<m^{*}(\beta)<1$.

2. When the book is thin, the follower submits a limit order for 1 round lot at price $A_{1}$ with probability $u_{T}^{*}=\frac{3}{4}$ and else does nothing. When the book is shallow, the follower adds 1 round lot at price $A_{1}$. When the book is deep, the follower does nothing.

3. The average small trade spread and the average large trade spread are greater than in the benchmark case.

The set of parameters for which this equilibrium is obtained is non-empty because (a) the condition $\beta<\beta^{*}$ implies that $m^{*}(\beta)<1$ and (b) the condition $\Delta<\alpha \sigma$ implies that $\beta^{*}>0$. This establishes that bluffing strategies can be sustained in equilibrium, even though they are correctly anticipated by the uninformed dealer. 
We now explain in detail the intuition behind the last proposition. The key point is that the state of the book contains information on the likelihood of an information event. When $m>0$, a large quoted spread has more chance to be observed when there is an information event than when there is not. ${ }^{19}$ Actually, the informed dealer chooses the large spread with probability 1 when there is an information event and with a smaller probability otherwise. Hence a large spread signals that an information event is impending. The quality of this signal increases with $m$. In fact if $\beta=0$ and $m=1$, a large spread is posted in the first stage only when an information event occurs and the signal is perfect. When $\beta>0$ and/or $m<1$, the size of the spread is an imperfect signal. Intuitively the quality of this signal increases with $m$ but it decreases with $\beta$. In particular, a large $\beta$ increases the likelihood that the best quotes have been set by a pre-committed trader and thereforethat they do not contain information.

For these reasons, when she observes a thin book (a large spread), the uninformed dealer revises upward the probability she assigns to an information event and the size of this revision increases with $m$ and decreases $\beta$. This is easily checked by computing $\pi_{T}(m, \beta)$, the uninformed dealer's posterior belief conditional on the book being thin at the end of stage $\mathrm{L}$ (for given values of $m$ and $\beta$ ). We obtain that

$$
\pi_{T}(m, \beta) \stackrel{\text { def }}{=} \operatorname{prob}(\Psi=1 \mid K=T)=\frac{\beta \Phi_{T}+(1-\beta)}{2 \beta \Phi_{T}+(1-\beta)(2-m)} \geq \pi_{0}=0 .
$$

Thus when she observes a large spread, the follower revises upward the probability she assigns to an information event and marks up the cost of liquidity provision. This reduces her incentive to submit a limit order at price $A_{1}$. We refer to this effect as being the deterrence effect. The larger is the follower's posterior belief $\left(\pi_{T}(m, \beta)\right)$, the larger is the deterrence effect. Thus the deterrence effect is strong when the quality of the signal provided by the spread is large ( $m$ small, $\beta$ large).

With these remarks in mind, we can now explain the nature of the equilibrium described in Proposition 3. Conditional on the state of the book being thin $(K=T)$, the uninformed dealer estimates the cost of offering one round lot at price $A_{1}$ to be :

$$
E_{\pi_{T}}(V \mid \widetilde{Q}(1) \geq 1)=v_{0}+\pi_{T}(m, \beta) \alpha \sigma .
$$

A graphical representation of this conditional expectation as a function of $m$ is given in Figure 3 . The perceived cost of offering 1 round lot at price $A_{1}$ for the uninformed dealer becomes larger as $m$ enlarges. This reflects the fact that the deterrence effect increases with $m$.

\section{INSERT FIGURE 3 ABOUT HERE}

\footnotetext{
${ }^{19}$ When $m=0$, the informed dealer does not bid differently when there is an information event and when there is not. Hence the offers at the end of stage $L$ are not informative.
} 
Observe on Figure 3 that $m^{*}(\beta)$ is the value of $m$ such that the follower is just indifferent between submitting a limit order for 1 round lot at price $A_{1}$ or doing nothing. That is $m^{*}(\beta)$ is such that:

$$
A_{1}-E_{\pi_{T}}(V \mid \widetilde{Q}(1) \geq 1)=\Delta-\pi_{T}\left(m^{*}, \beta\right) \alpha \sigma=0 .
$$

Suppose that the informed dealer chooses schedule $D$ with probability $m>m^{*}$. In this case a thin book induces a relatively large revision in the follower's estimation of the cost of liquidity provision. So large that she never finds it optimal to submit a limit order at price $A_{1}$ (see Figure 3 ). But then the informed dealer should choose to submit limit orders only at price $A_{2}$ (i.e he should always choose schedule $T$ ), whether an information event took place or not (i.e. $m=0$ ). This deviation precludes the existence of an equilibrium in which $m>m^{*}$. Suppose then that the informed dealer chooses schedule $D$ with probability $m<m^{*}$. In this case a thin book induces a relatively small revision in her estimation of the cost of liquidity provision by the follower. So small that she always finds it optimal to submit a limit order at price $A_{1}$. But then the informed dealer is strictly better off if he chooses schedule $D$ when there is no information event (i.e. $m=1$ ). This deviation precludes the existence of an equilibrium in which $m<m^{*}$.

When $m=m^{*}$, the follower is just indifferent between undercutting a thin book or doing nothing. Thus she follows a mixed strategy. She undercuts the thin book sometimes but not always. The leader is then confronted with a trade off between certain execution at price $A_{1}$ and uncertain execution at a more profitable price, $A_{2}$. In fact, when there is no information event, the informed dealer's expected profit if he establishes a thin book is:

$$
\Pi^{L}(T, 0) \stackrel{\text { def }}{=}\left(1-u_{T}\right)\left(A_{2}-v_{0}\right) E\left(\widetilde{Q}_{u}\right)+\frac{u_{T}}{2}\left(A_{2}-v_{0}\right)=\left(\left(1-u_{T}\right) \frac{3}{2}+\frac{u_{T}}{2}\right)\left(A_{2}-v_{0}\right),
$$

where $u_{T}$ is the probability that the follower undercuts the thin book with a limit order for 1 round lot at price $A_{1}$. In contrast, if the informed dealer chooses the deep book, he obtains an expected profit equal to

$$
\Pi^{L}(D, 0)=\frac{3\left(A_{1}-v_{0}\right)}{2} .
$$

It is immediate that the informed dealer is better off choosing a thin (resp.a deep) book iff $u_{T}<\frac{3}{4}$ (resp. $u_{T}>\frac{3}{4}$ ). For $u_{T}=\frac{3}{4}$, he is just indifferent and therefore he uses a mixed strategy, as described in the proposition.

These order placement strategies imply that the state of the book at the end of the bidding stage is random. For instance, suppose that the leader establishes a thin book. The follower reacts by improving upon the quotes with probability $\frac{3}{4}$ and does nothing otherwise. The book faced by market order submitters might then be shallow (with probability $\frac{3}{4}$ ) or thin (with probability 
$\left.\frac{1}{4}\right)$. Thus the book is not necessarily deep at date 1 , in contrast with the benchmark case. For this reason the liquidity of the market is smaller than in the benchmark case (last part of the proposition).

Observe that the informed dealer bids more aggressively when $\beta$ enlarges $\left(m^{*}(\beta)\right.$ increases with $\beta$ ). The intuition is as follows. Other things equal ( $m^{*}$ fixed), the size of the spread is less informative when $\beta$ increases. As we already explained, this relaxes the deterrence effect. Accordingly, in order to sustain the equilibrium with bluffing, the probability with which the informed dealer chooses schedule $D\left(m^{*}\right)$ must increase. This increase counterbalances exactly the effect of an increase in $\beta$ on the informativeness of the spread and the deterrence effect.

For $\beta$ large enough $\left(\beta>\beta^{*}\right)$, the follower cannot be deterred from submitting a limit order for 1 round lot at price $A_{1}$, even if $m=1$. In this case, there is no equilibrium in which the informed dealer uses a bluffing strategy. The equilibrium bidding strategies are described in the following proposition. Let $\beta^{* *} \stackrel{\text { def }}{=} \frac{((2-r) \alpha-r)}{((2-r) \alpha-r)+\Phi_{T}(2 r-(2-r) \alpha)}<1$.

Proposition 4 : When $\beta^{*}<\beta \leq \beta^{* *}$ and $\frac{2 \alpha \sigma}{\alpha+2}<\Delta<\alpha \sigma$, the following bidding strategies constitute an equilibrium:

1. When there is an information event, the informed dealer chooses schedule T. When there is no information event, the informed dealer chooses schedule D.

2. When the book is thin or shallow, the follower submits a limit order for 1 round lot at price $A_{1}$. When the book is deep, the follower does nothing.

3. The average small trade spread is as in the benchmark case but the average large trade spread is greater than in the benchmark case.

When she observes a thin book, the follower revises upward her belief regarding the likelihood of an information event. The revision is too small to deter her from submitting a limit order for 1 round lot at price $A_{1}$ but large enough to deter her from posting a larger size. In fact it is easily checked that :

$$
A_{1}-E_{\pi_{T}}(V \mid \widetilde{Q}(2) \geq 2)=\Delta-\left(\frac{2 \pi_{T}(1, \beta)}{\pi_{T}(1, \beta) \alpha+1}\right) \alpha \sigma \leq 0, \text { for } \beta \leq \beta^{* *},
$$

which means that the uninformed dealer perceives the cost of offering a second round lot at price $A_{1}$ as being larger than $A_{1}$ (see Figure 4 for $m=1$ ).

\section{INSERT FIGURE 4 ABOUT HERE}


The uninformed dealer bids more aggressively than in the equilibrium described in Proposition 3 but still more cautiously than in the benchmark case. This explains the last part of the proposition.

Proposition 5 : When $\beta>\beta^{* *}$ and $\frac{2 \alpha \sigma}{\alpha+2}<\Delta<\alpha \sigma$ then the following bidding strategies constitute an equilibrium:

1. When there is an information event, the informed deader chooses schedule T. When there is no information event, the informed deader chooses schedule D.

2. When the book is thin, the follower submits a limit order for 2 round lots. When the book is shallow, the follower submits a limit order for 1 round lot at price $A_{1}$ and when the book is deep, the follower does nothing.

3. The average small trade spread and the average large trade spread are as in the benchmark case.

Intuitively, when $\beta$ is very large (greater than $\beta^{* *}$ ), the size of the spread at the end of the intermediate bidding stage is not very informative. Actually there is a large probability that the spread has been established by traders without information. Hence the follower's belief about the occurence of an information event is not strongly affected by the orders placed in the book. Thus she behaves as in the benchmark case, that is she fills the book so that eventually 2 round lots are offered at price $A_{1}$. Anticipating this behavior, the leader establishes a deep book whenever this is profitable.

A Remark. In equilibrium, the follower's posterior belief about the occurrence of an information event is determined by Bayes rule whenever this is possible. As usual in signaling games, there is a difficulty if some states of the book are out-of-the equilibrium path. By definition these states have a zero probability of occurence in equilibrium. Hence in these states the follower's posterior belief cannot be determined by Bayes rule. This problem does not arise when $\beta>0$ (all states of the book are on the equilibrium path). When $\beta=0$, the shallow book is out-of the equilibrium path since the informed dealer never chooses a shallow book in the equilibria that we described previously. In this case, we make the conservative assumption that the follower does not revise her prior belief about the occurence of an information event when she observes a shallow book. ${ }^{20}$

\footnotetext{
${ }^{20}$ The Perfect Bayesian Equilibrium concept does not put restrictions on how players' beliefs should be formed when they observe actions that are out-of-the equilibrium path (actions which have a zero probability of occurence in equilibrium). For these actions, players' beliefs can be specified arbitrarily. See Fudenberg and Tirole (1991), Chapter 8.
} 


\subsection{The Non-Anonymous Limit Order Market}

In the non-anonymous market, we must consider two cases separately : (i) the leader is informed and (ii) the leader is uninformed. Actually, the optimal reaction of the follower is different in these two cases. The equilibrium in each case is readily obtained by considering polar cases of the analysis for the anonymous market. First, consider the polar situation in which $\beta=0$ in the anonymous market. In this case, the uninformed dealer knows that the leader is an informed dealer, even though she does not directly observe his identity. Accordingly the game in the anonymous market is identical to the game played in the non-anonymous market when the leader is informed. This remark yields the next corollary.

Corollary 1 : Consider the case in which the leader is the informed dealer. In this case, the dealers' bidding strategies described in Proposition 3 when $\beta=0$ form an equilibrium of the nonanonymous market. In particular, the informed dealer uses a bluffing strategy: when there is no information event, he chooses schedule $D$ with probability $m^{*}(0)<1$.

Now consider the other polar situation : $\beta=1$ in the anonymous market. In this case the uninformed dealer knows that the leader is a precommitted trader. Thus, the game in the anonymous market is identical to the game played in the non-anonymous market when the leader is a precommitted liquidity trader. We deduce that the equilibrium of the non-anonymous market when the leader is uninformed is identical to the equilibrium of the anonymous market when $\beta=1$. Hence it is described by Proposition 5 . As the limit orders posted in the first stage contain no information, the uninformed dealer optimally behaves as in the benchmark case. She fills the book so that 2 round lots are offered at price $A_{1}$ at the end of the bidding stage.

Anonymity and Bidding Aggressiveness. It is useful to analyze in detail how dealers' bidding behavior differs in the anonymous market and in the non-anonymous market. Ultimately this helps understanding how a switch to anonymity affects liquidity in our model. Observe that for a given value of $\beta$, the informed dealer chooses to establish a deep book with probability $m^{*}(\beta)$ in the anonymous market and probability $m^{*}(0)$ in the non-anonymous market, when there is no information event. Thus, as $m^{*}(\beta)>m^{*}(0)$, the informed dealer behaves more competitively in the anonymous market than in the non-anonymous market. Actually a switch to anonymity reduces the informational content of the quotes posted at the intermediate stage, other things equal. As explained in the previous section, this induces the informed dealer to post more aggressive limit orders.

The effect of anonymity on the uninformed dealer's bidding behavior is more complex. Consider the case in which the uninformed dealer faces a large spread (for the other states of the book, the uninformed dealer's behavior is not affected by the anonymity regime). In the non- 
anonymous market, the uninformed dealer undercuts the best offer with probability $u_{T}^{*}=\frac{3}{4}$ if the leader is informed and with probability 1 if the leader is a precommitted trader. Thus the probability of observing a limit order improving upon the large spread is:

$$
u_{T}^{*}(1-\beta)+\beta=\frac{(3+\beta)}{4},
$$

in the non-anonymous market. In the anonymous market, the uninformed dealer's behavior depends on his belief on the identity of the trader who set the large spread. If there is a large probability $\left(\beta \leq \beta^{*}\right)$ that this trader is an informed dealer, then the uninformed dealer behaves cautiously : he undercuts the best offer with probability $u_{T}^{*}=\frac{3}{4}$. In contrast, if there is a small probability $\left(\beta>\beta^{*}\right)$ that this trader is informed then the uninformed dealer is not deterred from improving upon the large spread : he places limit orders within the best quotes with probability 1 when the spread is large. As $\frac{3}{4}<\frac{(3+\beta)}{4}<1$, we conclude that the likelihood that the uninformed dealer improves upon a large spread can be smaller or larger in the anonymous market, depending on the value of $\beta$.

Another measure of the follower's aggressiveness is the probability that she will offer two round lots at price $A_{1}$ if she undercuts a large spread. This probability is $\beta$ in the non-anonymous market. In the anonymous market, this probability is equal to zero if $\beta \leq \beta^{* *}$ and 1 otherwise. Thus the follower can offer more or less depth at price $A_{1}$ in the anonymous market, depending on the value of $\beta$.

To sum up, the follower is more (resp. less) aggressive in the anonymous market if $\beta \geq \beta^{* *}$ (resp. $\beta \leq \beta^{*}$ ). For $\beta \in\left[\beta^{*}, \beta^{* *}\right]$, she undercuts the thin book more frequently in the anonymous market but with smaller orders than in the non-anonymous market.

\section{The Effects of a Switch to Anonymity}

In this section we compare measures of market liquidity in the anonymous and in the nonanonymous markets. Furthermore we study the informational content of the limit order book in the anonymous and in the non-anonymous market. In this way, we obtain several implications that we test in the next section.

\subsection{Anonymity and Market Liquidity}

We compute the equilibrium values of the small and the large trade spreads (as defined in Equations (5) and (6)) in the anonymous market and in the non-anonymous market. We obtain the following result. 
Corollary 2:A switch to an anonymous limit order book reduces the expected small and large trade spreads only when $\beta$ is large enough $\left(\beta \geq \beta^{* *}\right)$. When $\beta$ is small $\left(\beta<\beta^{*}\right)$, a switch to an anonymous limit order book enlarges the expected small and large trade spreads. When $\beta^{*}<\beta<\beta^{* *}$, a switch to anonymity: (i) reduces the expected small trade spread and (ii) increases the expected large trade spreads.

Thus a switch to an anonymous limit order book should affect liquidity. The impact, however is ambiguous and depends on $\beta$. Recall that the informed trader behaves more competitively in the anonymous market. However, when $\beta$ is small, the uninformed trader bids more conservatively (undercuts a thin book less frequently) in the anonymous market (see the previous subsection). These two effects have opposite impacts on market liquidity and the second effect dominates when $\beta$ is small. When $\beta$ is large enough, a switch to anonymity makes both the informed dealer and the uninformed dealer more aggressive. This explains why it reduces the small and the large trade spread.

Interestingly, for intermediate values of $\beta\left(\beta^{*}<\beta<\beta^{* *}\right)$, a switch to anonymity is beneficial to traders who submit small market orders (since it reduces the average small trade spread) but not to traders who submit large orders. Actually for these intermediate values the switch to anonymity reduces the probability that no round lots will be offered at price $A_{1}$ (i.e. $\operatorname{Pr}\left(Q_{1}=0\right)$ decreases). But, simultaneously, it reduces the probability that the uninformed dealer will offer 2 round lots at price $A_{1}$ (see previous subsection for an explanation). Overall the probability that 2 round lots will be offered at price $A_{1}$ (i.e. $\operatorname{Pr}\left(\widetilde{Q}_{1}=2\right)$ ) is smaller. Accordingly the probability that a large market order will walk up the book is larger and the large trade spread increases.

\subsection{Anonymity and the Informational Content of the Book}

There are two possible quoted spreads at the end of the bidding stage: Large $\left(A_{2}-v_{0}\right)$ or Small $\left(A_{1}-v_{0}\right)$ that we denote by " $L a$ " and "Sm" respectively. Notice that a large spread is observed at the end of the bidding stage only when the follower has chosen not to improve upon the quotes posted in stage 1 . Hence a large spread is always set by the leader. In contrast, a small spread at the end of the bidding stage may be set by the leader or by the follower. The informational content of the spread for future price volatility can be measured by

$$
\begin{aligned}
I_{B} & =E\left(\epsilon_{1}^{2} \mid \text { Spread }=L a\right)-E\left(\epsilon_{1}^{2} \mid \text { Spread }=\text { Sm }\right) \\
& =\sigma^{2}[\operatorname{Pr}(\Psi=1 \mid \text { Spread }=L a)-\operatorname{Pr}(\Psi=1 \mid \text { Spread }=S m)],
\end{aligned}
$$

where the second equality follows from the definition of $\epsilon_{1}$. The rationale for this measure is simple. If the size of the inside spread is non-informative then it should not help to forecast 
future price volatility (i.e. $E\left(\epsilon_{1}^{2} \mid\right.$ Spread $\left.=L a\right)=E\left(\epsilon_{1}^{2} \mid\right.$ Spread $\left.=S m\right)$ ). In this case $I_{B}=0$. In contrast if the size of the inside spread is informative then $I_{B} \neq 0$. In what follows, we denote by $I_{B}^{a}$ and $I_{B}^{n a}$ the informational content of the spread in the anonymous and in the non-anonymous markets, respectively.

Corollary 3 : In the non-anonymous market and in the anonymous market, the size of the bid-ask spread is informative about future price volatility : $I_{B}^{n a}>0$ and $I_{B}^{a}>0$. However the informational content of the bid-ask spread is smaller in the anonymous market, i.e. $I_{B}^{n a}>I_{B}^{a}$, when $\beta>\beta^{*}$.

We have argued in the previous section that a large spread signals that an information event is impending. Contrarily, a small spread signals the absence of an upcoming information event. This means that $I_{B}^{j}>0$ and this explains why the forecast of future price volatility increases with the size of the spread $\left(E\left(\epsilon_{1}^{2} \mid\right.\right.$ Spread $\left.=L a\right)>E\left(\epsilon_{1}^{2} \mid\right.$ Spread $\left.\left.=S m\right)\right)$.

The impact of a switch to anonymity on the informational content of the spread is complex. On the one hand, it reduces the incentive of an informed dealer to post a large spread when there is no information event $\left(m^{*}\right.$ increases with $\beta$ ). Hence his quotes are more sensitive to his information and thereby they contain more information on future volatility. On the other hand, the switch to anonymity can induce the non-informed dealer to establish a small spread more frequently (when $\beta \geq \beta^{*}$ ). Thus a small spread is less informative. For this reason, when $\beta \geq \beta^{*}$, the switch to anonymity reduces the informativeness of the bid-ask spread. Hence the forecast of future price volatility is less sensitive to the size of the spread (i.e. $E\left(\epsilon_{1}^{2} \mid \operatorname{Spread}=L a\right)-E\left(\epsilon_{1}^{2} \mid\right.$ Spread $=S m)$ ) is smaller in the anonymous market).

This corollary yields two new testable predictions. First, in time-series, the size of the spread in a given period should help to forecast the magnitude of price movements in subsequent periods (future price volatility). Furthermore the strength of the association between the size of the spread in one period and price volatility in a subsequent period should be affected by the anonymity regime. In particular, when a switch to anonymity reduces the spread on average $\left(\beta \geq \beta^{*}\right)$, the association between the size of the spread and subsequent price volatility should be weaker.

\subsection{Extensions}

More than 2 dealers. Our model of price formation in a limit order book is very stylised. Several of the results rely on the fact that the informed dealer uses a bluffing strategy in the non-anonymous environment and that his incentive to do so is reduced in the anonymous envi- 
ronment. A concern is that the incentive to bluff might disappear when the informed dealer faces competition from many uninformed dealers or from another informed dealer.

In order to study this question, in Appendix B, we consider an extension of the model in which : (i) the informed dealer competes with several uninformed dealers and (ii) the informed dealer competes with informed and uninformed dealers. In the first case, the equilibrium outcome is identical to the outcome obtained in the baseline model. In particular when $\beta \leq \beta^{*}$, the informed dealer uses the bluffing strategy described in Proposition 3. This is also the case when the follower may be informed if this does not happen with a too large probability (it must be smaller than 0.75). It is intuitive that this probability should not be too large. Actually, in the polar case in which it is equal to one, the informed dealer and the follower have symmetric information and therefore the situation is identical to the benchmark case.

Other Parameter Values. In the previous sections, we have analyzed in detail the equilibria which emerge when $\frac{2 \alpha \sigma}{\alpha+2}<\Delta<\alpha \sigma$. Analysis of other parameter values yields similar conclusions. In particular, consider the case in which $\alpha \sigma<\Delta<\frac{2 \alpha \sigma}{\alpha+1} .{ }^{21}$ In this case, it is profitable to offer one round lot (but no more) at price $A_{1}$ if there is an information event. Thus, the informed dealer posts a shallow book (rather than a thin book) when there is an information event. For $\beta$ small enough, the informed dealer uses a bluffing strategy : he sometimes posts the shallow book when there is no information event. In this case, this is not a large spread but rather a small quoted depth at price $A_{1}$ which signals that an information event is pending. But the implications are qualitatively identical to those we derived when $\Delta<\alpha \sigma$. In particular the lack of liquidity in the book foreshadows an informational event and the informativeness of the book is smaller in the anonymous market if $\beta$ is large enough. Furthermore a switch to anonymity decreases the large trade spread if $\beta$ is large enough. ${ }^{22}$

\footnotetext{
${ }^{21}$ The case in which $\Delta \geq \frac{2 \alpha \sigma}{\alpha+1}$ is not interesting. In this case, the tick size is so large that it is profitable to offer two round lots at price $A_{1}$ even if an information event occurs with probability one. Clearly, in this situation, the deterrence effect has no bite. Accordingly, there is no difference between the case in which dealers have asymmetric information and the case in which they have symmetric information.

${ }^{22}$ For these parameter values, the small trade spread is not affected by the switch to anonymity. But this is an artifact of the condition $\alpha \sigma<\Delta$. We have focused on the case $\Delta<\alpha \sigma$ to show that a switch to anonymity affects both the quoted spread and the quoted depth, in general.
} 


\section{Empirical Analysis}

\subsection{Institutional Background and Dataset}

\subsubsection{Euronext Paris}

In March 2000, the Amsterdam Stock Exchange, the Brussels Stock Exchange and the Paris Bourse decided to merge. This merger (which took place in September 2000) gave birth to Euronext, a holding with 3 subsidiaries: Euronext Amsterdam, Euronext Brussels and Euronext Paris. Since the merger, the 3 exchanges have strived to create a unique trading platform (called NSC). ${ }^{23}$ This goal is achieved since October 29, 2001. However, as of today, the 3 exchanges still have separate limit order books for each stock. Euronext Paris was first to adopt the new trading platform on April 23, 2001, soon followed by Brussels on May 21, 2001 and Amsterdam on October 29, 2001. ${ }^{24}$ For Euronext Paris, the trading rules were very similar before and after the switch to NSC. Indeed, for CAC40 stocks, the switch to an anonymous limit order book was the only significant change (see below).

NSC is an electronic limit order market (see Biais, Hillion and Spatt (1995) for a complete description of this market). Trading occurs continuously from 9:00 a.m. to 5:25p.m. for most of the stocks. ${ }^{25}$ The opening and the closing prices are determined by a call auction. All orders are submitted through brokers who trade for their own account or on behalf of other investors. Traders primarily use two types of orders: (a) limit orders and (b) market orders. Limit orders specify a limit price and a quantity to buy or to sell at the limit price. Limit orders are stored in the limit order book and executed in sequence according to price and time priority. If the limit price crosses a limit on the opposite side of the book (so called "marketable limit orders") then the limit order is immediately executed (entirely or partially depending on its size). Market orders execute upon arrival against the best price on the opposite side of the book. Any quantity in excess of the depth available at this price is transformed into a limit order at that price. Marketable limit orders can walk up or down the book (if they are large enough) whereas market orders do not (they can be viewed as marketable limit orders at the best price on the opposite side of the book).

All limit orders must be priced on a pre-specified grid. The tick size is a function of the stock price level. At the time of our study, the tick size is 0.01 Euros for prices below 50 Euros, 0.05 Euros for prices between 50.05 and 100 Euros, 0.1 Euros for prices between 100.1 Euros and 500

\footnotetext{
${ }^{23}$ NSC is an acronym which stands for "Nouveau Système de Cotation".

${ }^{24}$ The Lisboa Stock Exchange joined Euronext in 2002.

${ }^{25}$ Less liquid stocks trade in call auctions which take place at fixed points in time during the trading day. All stocks in our sample are traded continuously.
} 
Euros and 0.5 Euros for prices above 500 Euros. ${ }^{26}$

The transparency of the market is quite high. Brokers observe (on their computer terminals) all the visible limit orders (price and associated depth) standing in the book at any point in time. The 5 best limits on each side of the book, the total depth available at these limits and the number of orders placed at each limit are disclosed to the public. The depth available in the book can be larger than the visible depth. Actually NSC enables traders to display only a portion of their limit order by submitting hidden orders. The hidden portion retains price priority but loses time priority. A fraction of the hidden quantity becomes visible only when the quantity initially disclosed is fully executed.

Until April 23, 2001, but not after that date, the identification code of the issuing broker was also displayed for each order standing in the book. We refer to this change in the trading organization as the switch to anonymity. This switch applied to all stocks listed on Euronext Paris. The objective of market organizers was to harmonize the trading rules in Euronext Paris and Euronext Amsterdam (in which trading was anonymous). The electronic limit order book in the Paris Bourse had been non-anonymous since its inception in 1986. Interestingly, at this time, non-anonymity was viewed as a way to retain an essential feature of the open-outcry market used before the introduction of electronic limit order trading (see Muniesa (2003)).

Euronext Paris classifies stocks which trade continuously in 2 different groups, called "Continu A" and "Continu B". Stocks are assigned to one group based on measures of market activity (transaction and order frequency, trading volume). Stocks in Continu A feature a higher level of market activity. For stocks in Continu B, the switch to supply side anonymity was accompanied by another change. For these stocks, counterparty IDs used to be disclosed immediately after completion of their transaction until April 23, 2001. This is not the case anymore since this date. Thus stocks in Continu B have experienced a change in both pre-trade and post-trade anonymity. For this reason, it is difficult to isolate the effects of supply side anonymity on measures of market liquidity for these stocks. Fortunately, counterparty IDs have always been concealed for stocks in Continu A. Our empirical analysis uses CAC40 stocks. All the constituents stocks in this index belong to the Continu A group, and account for $84 \%$ of the total market capitalization of this group (at the time of our study).

\subsubsection{The Dataset}

The data (trades, quotes and orders) are obtained from the BDM database provided by Euronext Paris. Our dataset contains a time stamped record of all transactions and orders (price and

\footnotetext{
${ }^{26}$ In April 2001, the value of the euro in dollar was approximately 0.86 Dollar / Euro.
} 
quantities) submitted to the market from March 1 to May 30, 2001 for the constituent stocks of the CAC 40 index.

In order to avoid contamination of our findings due to the proximity of the event date, we drop two weeks of observations around April 23, 2001. We also drop all observations after May 20, 2001 in order to avoid confounding effects due to the adoption of NSC by Euronext Brussels. ${ }^{27}$ Finally we drop one stock from the sample because it was delisted from the index during the sample period. After these treatments, our data set contains 39 stocks and 28 trading days: (i) 14 trading days before the event from March 26 to April 12, 2001 and (ii) 14 trading days after the event from April 30 to May 20, 2001. We conduct our experiment on this dataset.

Additional but minor changes in trading rules took place for the stocks in our sample on April 23, 2001. Firstly, the Bourse changed some of the criteria which are used to select the opening price when there is a multiplicity of clearing prices at the opening. Secondly, it advanced by 5 minutes the end of the continuous trading session in order to facilitate the organization of the closing call auction. In our empirical analysis, we exclude observations collected during the first and the last 5 minutes of the continuous trading period. Thus our findings should not be contaminated by changes which affect the determination of opening and closing prices.

The Bourse also changed the treatment of orders which can trigger a trading halt. Trading halts occur when price changes exceed pre-specified thresholds. Before April 23, 2001 traders had the possibility to submit marketable limit orders resulting in a halt without partial execution of their order. Thus traders could suspend the trading process without bearing any direct cost. In contrast, as of April 23, 2001 marketable limit orders triggering a halt are partially executed up to the threshold price. This change in the handling of trading halts applied to all stocks. Hence there is no obvious way to control for its possible effects.

Table 3 presents some summary statistics (number of trades, average price, trading volumes, average trade sizes, daily return volatility and market capitalization) for our sample stocks. Separate figures are given for the pre-event period (March 26 to April 12) and the post-event period (April 30 to May 20). We further report t-values for a test for the equality of means and z-values for a Wilcoxon test for equality of medians.

The figures reveal a high level of trading activity for the stocks in our sample. The average daily number of transactions per stock is in the range of 2300. The number of transactions is slightly lower in the post-event period. On the other hand, the trading volume (in number of shares and in Euro) is higher in the post-event period. None of the differences are significant, however. Return volatility, defined as the standard deviation of 30 minute midquote returns,

\footnotetext{
${ }^{27}$ Arguably, this switch facilitated the access of Belgian traders to the French market. Thus, it may have increased the number of participants to Euronext Paris.
} 
is significantly lower in the post-event period. Thus, in our empirical analysis we will have to control for the possible effect of lower volatility on measures of market liquidity.

\subsection{Empirical Findings}

\subsubsection{Anonymity and Market Liquidity}

A first implication of our model is that measures of market liquidity such as the quoted spread and the effective spread should be different in the pre and in the post-event period. Furthermore the direction of the impact should be determined by the proportion of informed dealers, $(1-\beta)$ (see Section 5). It is difficult to design a direct test of the model because $\beta$ is not observed. Given our interpretations (see Section 3.1), a natural proxy for $\beta$ would be the proportion of agency limit orders. Unfortunately, in our dataset, we cannot identify principal and agency orders. For CAC40 stocks, Declerck (2001) finds that the 6 intermediaries which handled $71 \%$ of all principal trades accounted for only $39 \%$ of all orders during her study period. Furthermore, principal trading accounted for $27 \%$ of the trading volume, on average. These findings suggest that $\beta$ is relatively high for CAC40 stocks (which constitute our sample). Thus we expect a decrease in the quoted spread and in the effective spread after the switch to anonymity.

Univariate Analysis. We first calculate an average spread for each stock and each trading day. Then we average over the 14 days of the pre-event period and the 14 days of the postevent period. This results in two observations for each stock, one pre-event observation and one post-event observation. Finally, we average over the sample stocks.

We use two measures of the quoted bid-ask spread, namely, the quoted spread in Euro and the quoted percentage spread. ${ }^{28}$ We use two weighting schemes for computing these measures. The first gives each observation equal weight. The second assigns each observation a weight that corresponds to the time span during which the respective spread was valid. We thus have a total of four metrics for the effect of the switch to anonymity on the bid-ask spread.

The results are shown in Table 4 . We first observe that the different weighting schemes do not materially affect the spread estimates. Spreads in the post-event period are lower than those in the pre-event period. This holds irrespective of the spread measure used. The quoted spread in Euro has decreased by 0.03 Euro on average ( $21 \%$ of the average quoted spread in the pre-event period). We apply a t-test and a Wilcoxon test to investigate whether the reduction in the spread is significant. The test statistics, also shown in Table 4, indicate that the reduction is significant at the $5 \%$ level for the percentage quoted spread but not for the quoted spread in Euro. One

\footnotetext{
${ }^{28}$ In order to compute the quoted spread, we collect the value of the bid-ask spread each time there is a change in the size of the inside spread or in the quantities offered at the best quotes.
} 
potential explanation is that, as documented in Table 3, average prices were slightly higher in the period after the switch to anonymity. This reinforces the decrease in percentage spreads. Additionally, the minimum tick size is frequently binding for CAC40 stocks which prevents a decrease in the Euro spread. Hence, in the multivariate analysis, we will control for the effect of the price level and the tick size.

The effective spread is the absolute difference between the (average) price at which a market order executes and the quote midpoint prior to the trade multiplied by two (so that the effective spread is comparable to the quoted spread). This is a measure of price impact for a given trade. Formally :

$$
\text { Effective Spread }=2 *|P-m|,
$$

where $m$ is the quote midpoint 5 seconds prior to the transaction and $P$ is the transaction price. Some marketable limit orders exhaust the quantity offered at the best quotes and walk up or down the limit order book. These orders are reported as multiple trades occcuring at the same time at different prices in our dataset. Following Biais, Hillion and Spatt (1995), we classify these multiple trades as a single transaction at the weighted average price.

The last line in Table 4 reports the results. The average effective spread decreases from 0.154 Euro to 0.129 Euro. This decrease is, however, not statistically significant. This comparison does not take into account that the trade size distribution may have changed after the switch to anonymity. The trade size is a potential determinant of the effective spread since, at a given point in time, large orders should have larger price impact. Hence we estimate the average effective spread for each decile of trade sizes in our sample. We proceed as follows. First we classify transactions according to the trade size. Then, for each trade size decile, we calculate the average effective spread per stock and per trading day. ${ }^{29}$ Finally, we average over the 14 days of the pre- and the post-event period and finally aggregate over the sample stocks.

The results are presented in Figure 5. Overall, the effective spread has decreased for each trade size in our sample. There is no trade size for which the decrease is statistically significant, however. Observe that, in all but the largest order size classes, the effective spread is comparable to, or even smaller than, the average quoted spread. This indicates that liquidity demanders behave strategically : they submit their market orders when the spread is smaller than average.

The effective spread is indirectly a measure of the overall depth of the limit order book. Actually, the larger the quantities offered at given prices in the book, the smaller will be the price impact for a marketable order with a given trade size. Hence the previous results suggests

\footnotetext{
${ }^{29}$ Rule 11Ac1-5 by the SEC dictates that market centers in the U.S provide periodic measures of execution costs. The effective spread is one such measure. The SEC requires market centers to report this measure for different order size categories, as we do here.
} 
that the switch to anonymity has been accompanied by an increase in the depth of the book. We cannot formally test this hypothesis because we do not have data on the quantities offered behind the best quotes at a given point in time. However we can study the effect of the switch to anonymity on the number of shares offered at the best quotes (the "quoted depth").

Thus, in a third step, we analyze whether the quoted depth is significantly different after the switch to anonymity. It is well-known that quoted depth will tend to be larger, other things equal, at larger spreads (see Lee et al. (1993)). Hence we compare the quoted depth in the pre-event period and in the post-event period controlling for the level of the quoted spread. For each level of the quoted spread (between 1 and 9 ticks), we first calculate the average depth at the best bid and ask prices per stock and per trading day, then average over the 14 days of the pre- and the post-event period and finally aggregate over the sample stocks. Quoted depth can be measured in Euro or in number of shares. We obtain similar results in each case. For brevity we just report the findings for the quoted depth in Euro. ${ }^{30}$

The results are given in Figure 6. They indicate that the depth at the best quotes is larger in the post-event period for all nine quoted spread sizes. However, the change in quoted depth is generally not statistically significant.

Overall the results in this section indicate that the switch to anonymity has improved the liquidity of the market. The quoted spread and the effective spread have declined while the quoted depth is larger. This may explain why traders submit larger orders in the post-event period (see Table 3). Only the change in the relative quoted spread is statistically significant, however.

Multivariate Analysis. The changes in spreads and depth documented in the preceding section may be caused by variables we have not controlled for. In particular, Table 3 reveals that volatility is systematically lower in the post-event period. Furthermore we have pointed out that effects due to the tick size or changes in price levels between the two periods limit the conclusions which can be drawn from the univariate analysis. We use a regression framework to analyze whether the switch to anonymity affects spreads once we control for variables which are known to determine market liquidity.

Numerous empirical studies find that spreads depend on trading volume, the price level, and return volatility (see Stoll (2000)). We therefore include the log of the trading volume (in euro), the average price level and the standard deviation calculated from 30-minute midquote returns as control variables. As noted previously, the minimum tick size is a function of the price level of the stock. As the tick size potentially affects the size of the spread, we include the effective

\footnotetext{
${ }^{30}$ We collect the value of the quoted depth each time there is a change in the size of the inside spread or in the quantities offered at the best quotes.
} 
average tick size for stock $i$ as explanatory variable. It is defined as

$$
T S_{i, t}=\frac{1}{n} \sum_{j=1}^{n}\left[\frac{T S\left(A_{j}\right)+T S\left(B_{j}\right)}{2}\right],
$$

where $A_{j}\left[B_{j}\right]$ denotes the $j^{t h}$ ask (bid) price $(j=1, \ldots, n)$ observed on day $t$ and $T S($.$) denotes$ the minimum tick size associated with the ask and bid price, respectively. The tick size is a function of the stock price. It thus changes whenever a stock's bid and/or ask prices rises above, or falls below, one of the price thresholds which determine the tick size. The tick size can even be different on each side of the book if the ask and the bid price are above and below a threshold price, respectively. $T S_{i, t}$ is simply the average minimum tick size for stock $i$ on day $t$. To sum up, the regression model is

$$
s_{i, t}=\gamma_{0}+\gamma_{1} \log \left(\operatorname{Vol}_{i, t}\right)+\gamma_{2} T S_{i, t}+\gamma_{3} P_{i, t}+\gamma_{4} \sigma_{i, t}+\gamma_{5} D+\varepsilon_{i, t},
$$

where $s_{i, t}$ is a measure of the spread for firm $i$ at date $t, V o l_{i, t}$ is the trading volume for firm $i$ at date $t, T S_{i, t}$ is the average tick size for firm $i$ at date $t, P_{i, t}$ is the price level for firm $i$ at date $t, \sigma_{i, t}$ is the standard deviation of 30-minute midquote returns for firm $i$ at date $t$ and $D$ is a dummy variable which captures the effect of the switch to anonymity on the bid-ask spread (it takes on the value 1 for the observations in the anonymous regime). All variables are calculated for each stock and each day. We thus have one observation for each stock and each trading day.

We estimate separate regressions for the five spread measures described above (including the effective spread). The results are reported in Table 5 (under the label "Regression 1"). The independent variables explain a large part of the variation in bid-ask spreads, as evidenced by $R^{2}$ s ranging from 0.64 to 0.90 . All spread measures are negatively related to volume and are positively related to volatility. Quoted spreads measured in Euros and effective spreads are positively related to the price level whereas quoted percentage spreads are negatively related to the price level. Finally, we find a significant positive relation between the spreads and the effective tick size. This supports our conjecture that, for CAC40 stocks, the tick size may often be binding for the inside spread.

We now turn our attention to the effect of the post-event dummy. The coefficient on this variable is negative in each case, indicating that spreads are lower after the switch to anonymity. This confirms the findings of the univariate analysis. The results are even stronger because, for all spread measures, the reduction is statistically significant at the $5 \%$ level. The coefficient on the dummy variable indicates that the switch to anonymity has reduced the quoted spread by about 0.02 Euro (about $12 \%$ of the average quoted spread) and the effective spread by about 0.01 Euro.

We implemented a number of robustness checks. The regressions presented thus far assume that the intercept is constant across stocks. In a second set of regressions we allowed for different 
intercepts (i.e., fixed effects) by including stock-specific dummy variables. The results are also presented in Table 5 ("Regression 2"; we omitted the coefficients on the dummy variables to conserve space). Upon inclusion of the dummy variables the regression $R^{2}$ increases. The qualitative results remain unchanged.

A possible concern with our specification is that the error terms may be contemporaneously correlated across stocks because the switch to anonymity affects all stocks at the same time. To address that concern we implement a procedure proposed by Boehmer, Saar and Yu (2003). We include separate dummy variables for each day of the post-event period. The coefficient estimates are unbiased in the presence of contemporaneous correlation. Testing the median of the 14 dummies against zero provides a robust test of the hypothesis that spreads are lower in the post-event period. ${ }^{31}$ We also allow for stock-specific intercepts. Results are shown in the last 5 columns of Table 5 ("Regression 3"). The median of the dummy variables is always negative. In fact, in all cases all 14 individual dummies are negative. Therefore, the null hypothesis of a zero median is easily rejected. Overall, the multivariate analysis confirms the univariate results. The switch to anonymity is associated with lower bid-ask spreads.

\subsubsection{The Spread as a Signal of Future Price Changes.}

Now we turn to the model predictions regarding the informational content of the book on future (short-term) price volatility. Our purpose is twofold. First we want to test the hypothesis that the size of the spread contains information on the magnitude of future price movements. Second, we want to test the hypothesis that the switch to anonymity has altered the quality of the signal provided by the spread.

In order to perform these tests, we use the following methodology. For each stock in our sample, we partition each trading day into sixteen 30-minutes intervals and one 25 minutes interval (the last interval). We measure price volatility in interval $\tau \in\{1,2, \ldots, 17\}$ for stock $i$ by $V o l_{i \tau}=\left|m_{i \tau}-m_{i \tau-1}\right|$ where $m_{i \tau}$ is the midpoint of the best buyside and sellside limit prices at the end of interval $\tau$. Thus $V_{o l}$ is the magnitude of the price movement in interval $\tau$. Then we estimate the following pooled regression model :

$V o l_{i \tau+1}=a_{0}+a_{1} V o l_{M \tau+1}+a_{2} V o l_{i \tau}+a_{3} N_{i \tau}+a_{4} A T r_{i \tau}+\left(a_{5}+a_{6} D_{P o s t}\right) s_{i \tau}+\sum_{k=3}^{k=17} b_{k} T_{k \tau}+\sum_{i=2}^{k=39} c_{i} D_{i}+\varepsilon_{i \tau}$, where, for stock $i, N_{i \tau}$ is the number of transactions in interval $\tau, A T r_{i \tau}$ is the average trade size

\footnotetext{
${ }^{31}$ Another way to control for contemporaneous correlation (also proposed by Boehmer, Saar and Yu 2003) is to aggregate the data across stocks. This results in a time-series regression with 28 observations, one for each trading day. We estimated this model (results are not shown) and found the post-event dummy to be negative and significant. The results are thus fully consistent with those presented in the text.
} 
in interval $\tau, s_{i, \tau}$ is the average quoted spread in interval $\tau, D_{\text {Post }}$ is a dummy variable equal to 1 in the post event-period and zero in the pre-event period, $T_{k \tau}$ is a trading interval dummy equal to 1 if $k=\tau$ and the $D_{i}$ are stock-specific dummy variables allowing for different intercepts. We have partitioned each trading day into seventeen intervals but we only have sixteen intradaily observations per stock since we use lagged variables as independent variables. Furthermore we drop one trading interval dummy and one stock dummy to avoid linear dependence. $V_{\text {o }} l_{M \tau}$ is the market volatility, defined as the absolute change in the value of an equally weighted index of the sample stocks (calculated from quote midpoints) in interval $\tau$.

It is well-known that there are systematic intraday patterns in price volatility and that volatility is autocorrelated. We include the trading interval dummies $T_{k \tau}$ and the lagged volatility $V_{o l} l_{i \tau}$ in the list of independent variables to control for these effects. We also include the number of trades and the average trade size (in interval $\tau$ ) because Jones, Kaul and Lipson (1994) show that these variables are determinants of price volatility. The market volatility is included because the volatility for individual stocks depends on market conditions. Furthermore, the market volatility variable captures the decline in volatility from the pre-switch to the post-switch period documented above. ${ }^{32}$

Our regression model is similar in spirit to the model estimated by Ahn, Bae and Chan (2001). They use intraday data from the Hong-Kong Stock Exchange to study lead-lag relationships between quoted depth and price volatility. The focus of their paper however is very different from ours and they do not consider the spread as a potential determinant of future price volatility.

We are primarily interested in the effect of the quoted spread in a given period $\left(s_{i \tau}\right)$ on price volatility in the subsequent period $\left(V_{o l} l_{i \tau+1}\right)$. Recall that our hypothesis is that an increase in the spread in a given period foreshadows a large price movement in the subsequent period. Thus we expect $a_{5}>0$. Furthermore, the switch to anonymity should affect the sensitivity of the price volatility forecast to the spread. The findings of the previous section (smaller quoted and effective spreads after the switch to anonymity) are consistent with the model when $\beta>\beta^{*}$. Thus in this case we expect $a_{6}<0$ : the price volatility forecast is less sensitive to the size of the spread in the anonymous environment.

Table 6 reports the results. Consistent with our hypothesis, we find that the size of the spread in a given period is positively and significantly associated with price volatility in the subsequent period $\left(a_{5}=0.29\right)$. Furthermore, the sensitivity of the future price volatility to the size of the spread is significantly smaller in the post-event period $\left(a_{6}=-0.23\right)$. Overall the results support the view that (i) the size of the spread contains information about future price volatility and that

\footnotetext{
${ }^{32}$ Alternatively, we included the dummy variable $D_{P o s t}$ for the post-switch period. Its coefficient is negative and significant when the market volatility is excluded from the regression but changes sign and becomes insignificant once we include the market volatility. The other coefficients are not affected by these alternative specifications.
} 
(ii) its informativeness is smaller after the switch to anonymity.

We have performed a number of robustness checks (results not reported for brevity). ${ }^{33} \mathrm{We}$ checked that both the trading interval dummies and the stock-specific dummies are jointly signifiant. We added the contemporaneous volume variables (i.e., $N_{i \tau+1}$ and $A T r_{i \tau+1}$ ) to the model. The number of trades is significantly positively related to volatility. Inclusion of these variables does, however, not affect our main result. We further estimated separate regressions for the preswitch and the post-switch period. Consistent with our previous result we find that the spread is significantly and positively related to future volatility in both periods, but the relation is weaker in the post-switch period as is evidenced by a smaller coefficient estimate and a lower regression $R^{2}$.

Two possible concerns remain. First, our model assumes equal slope coefficients for all stocks. Second, there may be contemporaneous correlation among the residuals for different stocks. To address these concerns we estimated separate regressions for each stock. The residuals from these regressions are virtually uncorrelated. The mean of the 741 pairwise correlations is 0.002 and the highest correlation coefficient is less than $0.3 \cdot{ }^{34}$ Apparently, the market volatility variable included in the regression captures the co-movement in volatility. Our main result is also confirmed in these individual regressions, albeit somewhat weaker than in the pooled model. The coefficient on the lagged spread is positive in 38 out of 39 cases (and signifiant at the $10 \%$ level in 15 cases), the coefficient on the interaction term $D_{\text {Post }} s_{i \tau}$ is negative in 27 cases and significantly so in 8 cases. Among the 12 positive coefficients only one is significant.

In a final set of robustness checks we have estimated similar regression models using intervals of fifteen minutes instead of intervals of thirty minutes. Furthermore, we have considered the absolute or the squared return on the midquote as alternative measures of price volatility. With all these specifications, we obtain similar findings.

\subsubsection{Other Explanations}

Three empirical findings emerge : (i) the switch to anonymity has been followed by a reduction in bid-ask spreads, (ii) the size of the spread contains information on future price volatility but (iii) its informativeness has declined after the switch to anonymity. These findings are consistent with our model. Are there other possible explanations?

Simaan et al. (2003) argue that it is more difficult for liquidity providers to collude in an

\footnotetext{
${ }^{33}$ The results from the robustness checks are available upon request.

${ }^{34}$ Restricting the slope coefficients to be equal across stocks as is done in our base model yields similar results. The average correlation is 0.009 and the maximum value is 0.361 .
} 
anonymous environment. This hypothesis implies that a switch to anonymity will result in more competitive bid-ask spreads, as we find. However, collusion among liquidity suppliers is unlikely in a limit order market like Euronext because a large number of intermediaries compete in supplying liquidity. For instance, for the CAC40 stocks (our sample stocks), Declerk (2001) reports that they were 59 active broker-dealers in 1999. Furthermore this hypothesis does not explain why the informativeness of the bid-ask spread on future price volatility should be affected by the switch to anonymity.

Non-anonymity also facilitates the search of counterparties for block trading. For instance, consider an upstairs broker who must buy a block of shares for his client. Non-anonymity enables the broker to locate traders with large sell orders standing in the book. Then he can contact these traders directly (by phone) and arrange the trade without executing the order against the limit order book. If upstairs brokers were using brokers' IDs to this purpose then the switch to anonymity has increased their search costs. Hence it should reduce the volume of trades taking place upstairs. This reduction in market fragmentation may then result in a deeper limit order book. We call this the "search cost hypothesis".

In order to investigate further this hypothesis, we have computed the average daily number of block trades negotiated upstairs before and after the switch to anonymity. We also computed the number of block trades executed downstairs, that is, executed directly against the book. For each stock in our sample, Euronext Paris defines a "normal block size" (NBS). All orders larger than one NBS are considered as blocks and as such are eligible for special block trading rules. In particular, they can be negotiated upstairs and do not need to be executed at prices equal to or within the best bid and offer quotes. ${ }^{35}$ Hence we consider that a transaction is a block if its size exceeds one NBS. The NBS in our sample varies between 2,000 and 100,000 shares with an average value of 19410.26 shares. If the "search cost hypothesis" is correct, the number of upstairs trades should decrease and the number of downstairs trades should increase, after the switch to anonymity.

Table 7 reports the results. The average daily number of upstairs trades has decreased after the switch to anonymity (from about 4 trades per day to 2.4 trades). The decrease is significant at the $5 \%$ level. The average daily trading volume negotiated upstairs also has decreased, but not significantly. The number and the volume of downstairs trades have increased but again these changes are not significant. Overall these mixed results are not very supportive of the "search cost hypothesis". We also note that this hypothesis cannot explain why the informativeness of the spread on future price volatility has changed after the switch to anonymity.

\footnotetext{
${ }^{35}$ See Bessembinder and Venkataraman (2003) for a detailed analysis of block trading on Euronext Paris.
} 


\section{Conclusions}

We consider a model of limit order trading in which some limit order traders have superior information on the likelihood of future price movements. Informed limit order traders bid more conservatively when they expect a large price movement (an information event). For this reason, the state of the book is informative about future price movements. In particular a large bidask spread signals an impending price movement and thereby it reduces uninformed dealers' inclination to improve upon posted offers. Informed dealers exploit this effect to earn larger rents by using bluffing strategies : sometimes, they set large spreads while they could profitably set more competitive quotes.

We show that these strategic interactions imply that the amount of information provided on limit order traders' IDs affects market liquidity. When these IDs are concealed, uninformed dealers' bidding strategies are determined by their belief on the type of the trader setting the best quotes (informed/uninformed). If there is a large probability that a large spread is set by an informed dealer, they do not improve upon the quotes while if this probability is small, they improve upon the best quotes. In contrast, when limit order traders' IDs are disclosed, uninformed dealers' bidding strategy is determined by the actual type of the trader setting the best quotes. It follows that uninformed dealers can behave more or less aggressively in the anonymous market, depending on the fraction of informed traders in the population of liquidity suppliers. As for informed limit order traders, they always bid more aggressively in the anonymous market. Overall, these results imply that a switch to anonymity results in smaller trading costs for small and large orders when the fraction of informed limit order traders is small.

We also show that concealing limit order traders' IDs alters the informational content of the book. On the one hand, in the anonymous market, informed dealers post quotes which reflect more faithfully their private information on the magnitude of future price movements. This effect increases the informational content of the book in the anonymous regime. On the other hand, when the fraction of informed limit order traders is small, uninformed dealers play a more active role in setting the best quotes in the anonymous regime. As their orders do not contain information, this effect works to reduce the informational content of the book when limit order traders' IDs are concealed. On balance, we find that concealing limit order traders' IDs reduces the informational content of the book for parameter values for which it reduces the quoted spread.

Thus, a switch to anonymity has an impact on market liquidity and the informational content of the limit order book but the direction of this impact is an empirical question. On April 23, 2001, the limit order book for stocks listed on Euronext Paris became anonymous. We compare spreads and quoted depth before and after this event for a sample of 39 actively traded stocks. This natural experiment indicates that quoted and effective spreads are significantly smaller 
in the anonymous market. Moreover we find that the quoted depth has increased (albeit not significantly). Overall the results suggest that the switch to anonymity has improved market liquidity.

We also study the intraday relationship between price volatility and the size of the bid-ask spread. We divide each trading day in intervals of thirty minutes. We find that there is a positive and significant relationship between the magnitude of the price movement in one period and the size of the spread in the previous period. The association is weaker (albeit still significant) after the switch to anonymity. This is consistent with a smaller informativeness of the spread after the switch to anonymity. There are other possible explanations for the impact of anonymity on the bid-ask spreads. But, unlike our model, these alternative explanations fail to explain why the switch to anonymity also affects the informativeness of the spread for future volatility.

Our findings suggest several interesting venues for future research. The logic our model suggests that a lack of liquidity in the book foreshadows a price movement. This lack of liquidity manifests itself by a large spread but also by a steeper book. This suggests that the slope of the book, in addition to the size of the spread, may also contain information on future price volatility. ${ }^{36}$ This could be tested with more detailed data. On another front, the analysis raises intriguing questions about the relationships between changes in option prices and the liquidity of the underlying securities. Options contain information on the price volatility of the underlying security (see Lamoureux and Lastrape (1993) or Szakmary et al.(2003)). How does this information affect limit order prices in the market for the underlying security? Converserly, how does information on future price volatility contained in the limit order book affect option prices?

\section{References}

[1] Albanesi, S. and Rindi, B. (2000): "The Quality of the Italian Treasury Bond Market, Asymmetric Information and Transaction Costs", Annales d'Economie et Statistique, 60, 1-19.

[2] Anand, A. and Martell, T. (2001): "Informed Limit Order Trading", mimeo, Syracuse University.

[3] Baruch, S. (2002): "Who Benefits from an Open Limit Order Book", Working Paper, University of Utah.

\footnotetext{
${ }^{36}$ Naes and Skjeltorp (2003) find empirically a negative relationship between volatility and the depth of the book. Their results however are not directly comparable to ours because they analyze the comtemporaneous (instead of the lagged) relationship between volatility and the depth of the book at the daily frequency (instead of considering intraday relationships).
} 
[4] Benveniste, L., Marcus, A. and Wilhelm, W. (1992): "What 's Special about the Specialist?", Journal of Financial Economics, 32, 61-86.

[5] Bessembinder, H. and Venkataraman, K. (2003): "Does an Electronic Exchange Need and Upstairs Market?", forthcoming Journal of Financial Economics.

[6] Biais, B. Hillion, P. and Spatt, C. (1995): "An Empirical Analysis of the Limit Order Book and the Order Flow in the Paris Bourse", Journal of Finance, 50, 1655-1689.

[7] Bloomfield, R., O'Hara, M. and Saar, G. (2003) : "The 'Make' or 'Take' Decision in an Electronic Market : Evidence on the Evolution of Liquidity", forthcoming Journal of Financial Economics.

[8] Boehmer, E. Saar, G. and Yu, L. (2003): "Lifting the Veil: An Analysis of Pre-Trade Transparency at the NYSE", mimeo, NYSE and NYU.

[9] Calcagno, and Lovo, S. (2001): "Market Efficiency and Price Formation when Dealers are Asymmetrically Informed", Working Paper, HEC School of Management.

[10] Cao, C., Hansch, O. and Wang, X. (2003): "The Informational Content of an Open Limit Order Book", working paper, Pennsylania State University.

[11] Copeland, T. and Galai, D. (1983): "Information Effects and the Bid-Ask Spreads", Journal of Finance, 38, 1457-1469.

[12] Declerk, F. (2001): "Dual Trading and Order-Driven Market Liquidity: An Empirical Analysis of Euronext Members' Principal Trading", Working Paper, Toulouse University.

[13] Easley, D. and O'Hara, M. (1992): "Time and the Process of Security Price Adjustment", Journal of Finance, 57,577-605.

[14] Foucault, T., Roëll, A. and Sandas, P. (2002): "Market-Making with Costly Monitoring: An Analysis of the SOES Controversy", Review of Financial Studies, 16, 345-384.

[15] Foucault, T., Kandel, E. and Kadan, O. (2003): "Limit Order Book as a Market for Liquidity", Working Paper, CEPR.

[16] Forster, M. and Georges, T. (1992): "Anonymity in Securities Market", Journal of Financial Intermediation, 2, 168-206.

[17] Fudenberg, D. and Tirole, J. (1991), Game Theory, MIT Press.

[18] Garfinkel, J. and Nimalendran, N. (2002): "Market Structure and Trader Anonymity: An Analysis of Insider Trading", forthcoming Journal of Financial and Quantitative Analysis. 
[19] Glosten, L. (1994): "Is the Electronic Limit Order Book Inevitable", Journal of Finance, 49, 1127-1161.

[20] Harrington, J. (1987): "Oligopolistic Entry Deterrence under Incomplete Information", Rand Journal of Economics, 18, 211-231.

[21] Harris, L. and Hasbrouk, J. (1996): "Market vs. limit Orders: the SuperDot Evidence on Order Submission Strategy", Journal of Financial and Quantitative Analysis, 31, 213-231.

[22] Harris, L. and Panchapagesan, V. (2003): "The Information Content of the Limit Order Book : Evidence from NYSE Specialist Decisions", Working Paper, USC.

[23] Ahn, H., Bae, K. and Chan, K. (2001):"Limit Orders, Depth, and Volatility : Evidence from the Stock Exchange of Hong Kong", Journal of Finance, 56, 769-790.

[24] Hellwig, M. (1980): "On the Aggregation of Information in Competitive Markets", Journal of Economic Theory, 22, 477-498.

[25] Irvine, P., Benston, P. and Kandel, E. (2000) : "Liquidity beyond the Inside Spread: Measuring and Using Information in the Limit Order Book", Working Paper, Emory University.

[26] Kalay, A. and Wohl, A. (2002): "The Information Content of the Demand and Supply Schedules of Stocks", mimeo, Tel Aviv University.

[27] Lamoureux, C. and Lastrapes, W. (1993): "Forecasting Stock-Return Variance: Toward an Understanding of Stochastic Implied Volatilities", Review of Financial Studies, 6, 293-326.

[28] Lee, C., Mucklow, B. and Ready, M. (1993): "Spreads, Depths and the Impact of Earnings Information: An Intraday Analysis", Review of Financial Studies, 6, 345-374.

[29] Madhavan, A. and Cheng, M. (1997): "In search of liquidity: block trades in the upstairs and downstairs markets", Review of Financial Studies, 10, 175-203.

[30] Madhavan, A., Porter, D. and Weaver, D. (2002): "Should Securities Markets be Transparent", Working Paper, Baruch College.

[31] Milgrom, P. and Roberts, J. (1982): "Limit Pricing and Entry under Incomplete Information: an Equilibrium Analysis", Econometrica, 50, 443-459.

[32] Muniesa, F. (2003), Markets as Algorithms: The Sociology of Exchange Automation at the Paris Bourse, Ph.D thesis, Ecole des Mines de Paris.

[33] Naes, R. and Skjeltorp, A. (2003) : "Order Book Characteristics and the Volume-Volatility Relation : Empirical Evidence from a Limit Order Market", mimeo, Norwegian School of Economics and Business Administration. 
[34] O’Hara, M. (1995), Market Microstructure Theory, Blackwell.

[35] Rindi, B. (2002), "Transparency, Liquidity and Price Formation", Working Paper, Bocconi University.

[36] Roth, A. and Ockenfels, A. (2002), "Last-Minute Bidding and the Rules for Ending SecondPrice Auctions: Evidence from eBay and Amazon Auctions on the Internet", forthcoming American Economic Review.

[37] Szakmary, A., Ors, E., Kim, J. and Davidson, W. (2003): "The Predictive Power of Implied Volatility : Evidence from 35 Futures Markets", Journal of Banking and Finance, 27, 21512175 .

[38] Sandås, P., (2000), "Adverse Selection and Competitive Market Making: Evidence from A Limit Order Market", Review of Financial Studies, 14, 705-734.

[39] Seppi, D. (1990): "Equilibrium block trading and asymmetric information", Journal of Finance, 45, pp 73-94.

[40] Seppi, D. (1997): "Liquidity-Based Competition for Order Flow", Review of Financial Studies, 11, 789-816.

[41] Simaan, Y., Weaver, D. and Whitcomb, D. (2003): "Market Maker Quotation Behavior and Pre-Trade Transparency", Working Paper, Baruch College.

[42] Stoll, H. (2000): "Friction", Journal of Finance, 1479-1514.

[43] Theissen, E. (2003): "Trader anonymity, price formation and liquidity", European Finance Review, 7, 1-26.

[44] Waisburd, A. (2003): "Anonymity and Liquidity: Evidence from the Paris Bourse", mimeo, Texas Christian University. 
Table 1: Main Notations

\begin{tabular}{||l|l|}
\hline$\widetilde{V}_{2}$ & Final value of the security at Date 2 \\
\hline$\epsilon_{1}$ & Innovation at date 1 \\
\hline$v_{0}$ & Unconditional expected value of the security \\
\hline$\alpha$ & Probability of order submission by a speculator if information event \\
\hline$q$ & Size of 1 round lot \\
\hline$\pi_{0}$ & Prior probability of an information event \\
\hline$\sigma$ & Size of an innovation \\
\hline$\beta$ & Probability that the leader is a precommited trader \\
\hline$\Delta$ & Tick size \\
\hline$A_{j}$ & $j^{\text {th }}$ ask price on the grid above the unconditional expected value \\
\hline$K$ & State of the book at the end of the first stage \\
\hline$\Phi_{K}$ & Probability that the state of the book is K if the leader is a pre-commited trader \\
\hline$Q_{1}$ & Depth of the book at price $A_{1}$ \\
\hline$Q_{s}$ & Size of the market order submitted by a speculator \\
\hline$Q_{l}$ & Size of the market order submitted by a liquidity trader \\
\hline$\pi_{K}$ & Follower's belief about the occurence of an information event \\
\hline$\Psi$ & Indicator variable (0 if there is no information event; 1 otherwise) \\
\hline
\end{tabular}




\section{Appendix}

\subsection{Appendix A}

Preliminary Remarks. Let $\Pi^{F}(n, K)$ be the follower's expected profit if she offers $n$ round lots at price $A_{1}$ conditional on the state of the book being $K$ at the end of stage $L$ and conditional on the arrival of a buy order at date 1 (this is the expected profit on a sell limit order). Obviously $\Pi^{F}(0, K)=0$. Furthermore, we have

$$
\Pi^{F}(2, T)=\left[\pi_{T}\left[2 \alpha\left(A_{1}-\left(v_{0}+\sigma\right)\right)+\frac{3}{2}(1-\alpha)\left(A_{1}-v_{0}\right)\right]+\frac{3}{2}\left(1-\pi_{T}\right)\left(A_{1}-v_{0}\right)\right],
$$

which rewrites (using the expressions for $E_{\pi_{T}}\left(V \mid \widetilde{Q}(1) \geq 1\right.$ ) and $E_{\pi_{T}}(V \mid \widetilde{Q}(2) \geq 2)$ given in Equations (8) and (10)):

$$
\Pi^{F}(2, T)=A_{1}-E_{\pi_{T}}(V \mid \widetilde{Q}(1) \geq 1)+\operatorname{Pr}(\widetilde{Q}(2) \geq 2 \mid K=T)\left(A_{1}-E_{\pi_{T}}(V \mid \widetilde{Q}(2) \geq 2)\right),
$$

where $\operatorname{Pr}(\widetilde{Q}(2) \geq 2 \mid K=T)=\frac{\alpha \pi_{T}+1}{2}$. Using the same type of reasoning we also obtain:

$$
\Pi^{F}(1, T)=A_{1}-E_{\pi_{T}}(V \mid \widetilde{Q}(1) \geq 1)
$$

and

$$
\Pi^{F}(1, S)=\operatorname{Pr}(\widetilde{Q}(2) \geq 2 \mid K=S)\left(A_{1}-E_{\pi_{S}}(V \mid \widetilde{Q}(2) \geq 2)\right)
$$

These expressions will be used in the proofs below.

Proof of Lemma 1. The proof follows directly from the arguments in the text. The reader can also check the claim by using the follower's expected profits given in Equations (26), (27) and (28)

Proof of Proposition 1. It follows from the argument before the proposition.

Proof of Proposition 2. We denote by $\Pi^{L}(K)$, the leader's expected profit if he posts schedule $K$ conditional on the arrival of a buy order at date 1 . The follower's reaction is given in Lemma 1 for $\pi_{S}=\pi_{T}=0.5$ (since dealers have symmetric information). It follows that the book at the end of the bidding stage will be deep (since $\frac{2 \alpha \sigma}{\alpha+2}<\Delta$ ). Given the follower's reaction, we deduce that

$$
\begin{gathered}
\Pi^{L}(T)=0, \\
\Pi^{L}(S)=\pi_{0}\left[\alpha\left(A_{1}-\left(v_{0}+\sigma\right)\right)+(1-\alpha)\left(A_{1}-v_{0}\right)\right]+\left(1-\pi_{0}\right)\left(A_{1}-v_{0}\right)=A_{1}-E_{\pi_{0}}(V \mid \widetilde{Q}(1) \geq 1),
\end{gathered}
$$




$$
\Pi^{L}(D)=\pi_{0}\left[2 \alpha\left(A_{1}-\left(v_{0}+\sigma\right)\right)+\frac{3}{2}(1-\alpha)\left(A_{1}-v_{0}\right)\right]+\frac{3}{2}\left(1-\pi_{0}\right)\left(A_{1}-v_{0}\right),
$$

which rewrites (using the expressions for $E_{\pi_{0}}\left(V \mid \widetilde{Q}(1) \geq 1\right.$ ) and $E_{\pi_{0}}(V \mid \widetilde{Q}(1) \geq 2)$ ):

$$
\Pi^{L}(D)=A_{1}-E_{\pi_{0}}(V \mid \widetilde{Q}(1) \geq 1)+\operatorname{Pr}(\widetilde{Q}(2) \geq 2)\left(A_{1}-E_{\pi_{0}}(V \mid \widetilde{Q}(2) \geq 2)\right),
$$

where $\operatorname{Pr}(\widetilde{Q}(2) \geq 2)=\frac{\alpha \pi_{0}+1}{2}$ is the probability that a buy order at date 2 is larger than 2 round lots (when 2 round lots are offered at price $A_{1}$ ). Condition (14) implies that

$$
E_{\pi_{0}}(V \mid \widetilde{Q}(2) \geq 2)=v_{0}+\frac{2 \pi_{0} \alpha \sigma}{\pi_{0} \alpha+1}=v_{0}+\frac{2 \alpha \sigma}{\alpha+2}<v_{0}+\Delta .
$$

Furthermore we know that $E_{\pi_{0}}(V \mid \widetilde{Q}(1) \geq 1)<E_{\pi_{0}}(V \mid \widetilde{Q}(2) \geq 2)$. Therefore we conclude that

$$
E_{\pi_{0}}(V \mid \widetilde{Q}(1) \geq 1)<E_{\pi_{0}}(V \mid \widetilde{Q}(2) \geq 2)<A_{1} .
$$

It immediately follows that

$$
\Pi^{L}(T)<\Pi^{L}(S)<\Pi^{L}(D),
$$

which proves that the dealer acting in stage $L$ chooses schedule $D$.

\section{Proof of Proposition 3.}

Step 1. We show that the follower's bidding strategy is a best response to the informed dealer's bidding strategy. First consider the case in which the book is thin at the end of the first stage. The follower's expected profit if she submits a limit order for 1 round lot at price $A_{1}$ is (see Eq. (27) in the preliminary remarks):

$$
\Pi^{F}(1, T)=A_{1}-E_{\pi_{T}}(V \mid \widetilde{Q}(1) \geq 1)
$$

that is (using Equation (8)):

$$
\Pi^{F}(1, T)=\Delta-\alpha \pi_{T}\left(m^{*}, \beta\right) \sigma
$$

Substituting $m^{*}(\beta)$ by its expression in $\pi_{T}\left(m^{*}, \beta\right)$ (given by Eq.(17)) and then substituting $\pi_{T}\left(m^{*}, \beta\right)$ in Equation $(29)$, we find that

$$
\Pi^{F}(1, T)=\Delta-\alpha \pi_{T}\left(m^{*}, \beta\right) \sigma=0 .
$$

Furthermore Equation (26) yields

$$
\Pi^{F}(2, T)=A_{1}-E_{\pi_{T}}(V \mid \widetilde{Q}(1) \geq 1)+\operatorname{Pr}(\widetilde{Q}(2) \geq 2 \mid K=T)\left(A_{1}-E_{\pi_{T}}(V \mid \widetilde{Q}(2) \geq 2)\right) .
$$

As $A_{1}=E_{\pi_{T}}(V \mid \widetilde{Q}(1) \geq 1)$ (as we just have shown) and because $\left.E_{\pi_{T}}(V \mid \widetilde{Q} \geq 2)\right)>E_{\pi_{T}}(V \mid$ $\widetilde{Q} \geq 1)$, we deduce that $\Pi^{F}(2, T)<0$. Hence we have shown that:

$$
\Pi^{F}(1, T)=\Pi^{F}(0, T)>\Pi^{F}(2, T) .
$$


Thus, when she observes a thin book, the follower's optimal reaction is either to submit a limit order for 1 round lot or to do nothing. As she is indifferent, the mixed strategy given in the proposition is a best response for the follower. In equilibrium, the informed dealer never chooses a shallow book (whether $\Psi=1$ or not). Thus when she observes a shallow book, the follower does not update her beliefs and behaves as in the benchmark case. ${ }^{37}$ These arguments establish the second part of the proposition.

Step 2. We show that the informed dealer's bidding strategy is a best response. We denote by $\Pi^{L}(K, \Psi)$, the leader's expected profit in state $\Psi$ if he posts schedule $K$ conditional on the arrival of a buy order at date 1 . When $\Psi=0$, straightforward computations yield (taking into account the follower's reaction):

$$
\Pi^{L}(T, 0)=\left(1-u_{T}^{*}\right)\left(A_{2}-v_{0}\right) E\left(\widetilde{Q}_{u}\right)+\frac{u_{T}^{*}}{2}\left(A_{2}-v_{0}\right)=\left(1-u_{T}^{*}\right)\left(A_{2}-v_{0}\right) \frac{3}{2}+\frac{u_{T}^{*}}{2}\left(A_{2}-v_{0}\right) .
$$

and

$$
\Pi^{L}(S, 0)=A_{1}-v_{0}
$$

and

$$
\Pi^{L}(D, 0)=E\left(\widetilde{Q}_{u}\right)\left(A_{1}-v_{0}\right)=\frac{3}{2}\left(A_{1}-v_{0}\right) .
$$

Using the fact that $u_{T}^{*}=\frac{3}{4}$, we obtain

$$
\Pi^{L}(D, 0)=\Pi^{L}(T, 0)>\Pi^{L}(S, 0)
$$

Thus when $\Psi=0$, the leader optimally chooses schedule $D$ or schedule $T$. As she is indifferent between these two schedules, choosing schedule $D$ with probability $m^{*}(\beta)$ and schedule $T$ with probability $\left(1-m^{*}(\beta)\right)$ is a best response. Notice that $m^{*}(\beta)<1$ if $\beta<\beta^{*}$.

Now we consider the informed dealer's optimal reaction when $\Psi=1$. Given the follower's reaction and the informed trader's behavior, we deduce that:

$$
\Pi^{L}(T, 1)=(1-\alpha)\left[\left(1-u_{T}^{*}\right)\left(A_{2}-v_{0}\right) \frac{3}{2}+\frac{u_{T}^{*}}{2}\left(A_{2}-v_{0}\right)\right]>0 .
$$

and

$$
\Pi^{L}(S, 1)=A_{1}-E_{1}(V \mid \widetilde{Q}(1) \geq 1)
$$

and

$$
\Pi^{L}(D, 1)=A_{1}-E_{1}(V \mid \widetilde{Q}(1) \geq 1)+\operatorname{Pr}(\widetilde{Q}(2) \geq 2)\left(A_{1}-E_{1}(V \mid \widetilde{Q}(2) \geq 2)\right)
$$

\footnotetext{
${ }^{37}$ The informed dealer never chooses a shallow book. Thus when $\beta=0$, the probability of observing a shallow book at the end of the first stage of the bidding stage is zero. The follower's posterior belief after observing a shallow book cannot be computed by bayes rule in this case. In this case (see remark at the end of Section 4.2), we assume that the follower's belief on the occurence of an information event is given by her prior belief. This guarantees continuity with respect to $\beta$ of the follower's posterior belief conditional on observing a shallow book.
} 
Using Eq.(8) and (10), we obtain $E_{1}(V \mid \widetilde{Q}(1) \geq 1)=v_{0}+\alpha \sigma$ and $E_{1}(V \mid \widetilde{Q}(2) \geq 2)=v_{0}+\frac{2 \alpha \sigma}{\alpha+1}$. Hence when $\Delta \leq \alpha \sigma$, we have

$$
A_{1} \leq E_{1}(V \mid \widetilde{Q}(1) \geq 1)<E_{1}(V \mid \widetilde{Q}(2) \geq 2) .
$$

Hence, we deduce that

$$
\Pi^{L}(T, 1)>0>\operatorname{Max}\left\{\Pi^{L}(S, 1), \Pi^{L}(D, 1)\right\}
$$

Thus when $\Psi=1$, the leader optimally chooses schedule $T$.

Finally observe that there cases in which the book will be thin at the end of the bidding stage. This happens when (i) the informed dealer chooses a thin book and the follower does not undercut or (ii) a pre-commited trader establishes a thin book and the follower does not undercut. Thus there are cases in which large or small orders will execute at price $A_{2}$. In the benchmark case, all orders execute at price $A_{1}<A_{2}$. This remark yields the last part of the proposition

\section{Proof of Proposition 4.}

Part 1. We first show that the follower's bidding strategy of the follower is a best response. First consider the case in which the book is thin. The follower's expected profit if she submits a limit order for 1 round lot at price $A_{1}$ is (using Equation (29) in the proof of the previous proposition):

$$
\Pi^{F}(1, T)=\Delta-\alpha \pi_{T}(1, \beta) \sigma
$$

Given the informed dealer's bidding behavior, bayesian calculus yields:

$$
\pi_{T}(1, \beta)=\operatorname{prob}(\Psi=1 \mid K=T)=\frac{\beta \Phi_{T}+(1-\beta)}{2 \beta \Phi_{T}+(1-\beta)} .
$$

It is then easily checked that

$$
\Pi^{F}(1, T)=\Delta-\alpha \pi_{T}(1, \beta) \sigma=\Delta-\alpha\left[\frac{\beta \Phi_{T}+(1-\beta)}{2 \beta \Phi_{T}+(1-\beta)}\right] \sigma>0,
$$

iff $\beta^{*}<\beta$. Furthermore the follower's expected profit if she submits a limit order for 2 round lots (given that the book is thin) can be written (see Equation (26)):

$$
\Pi^{F}(2, T)=\Pi^{F}(1, T)+\operatorname{Pr}(\widetilde{Q}(2) \geq 2 \mid K=T)\left(A_{1}-E_{\pi_{T}}(V \mid \widetilde{Q}(2) \geq 2)\right) .
$$

Recall that

$$
A_{1}-E_{\pi_{T}}(V \mid \widetilde{Q}(2) \geq 2)=\Delta-\left(\frac{2 \pi_{T}(1, \beta)}{\pi_{T}(1, \beta) \alpha+1}\right) \alpha \sigma .
$$


It is easily checked that $\pi_{T}(1, \beta)$ is such that

$$
A_{1}-E_{\pi_{T}}(V \mid \widetilde{Q}(2) \geq 2) \leq 0
$$

iff $\beta \leq \beta^{* *}$. Thus, if $\beta \leq \beta^{* *}$, the follower never submits a limit order for two round lots at price $A_{1}$ since she expects to lose money on the second round lot. Hence we have shown that the follower's best response when the book is thin is to submit a limit order for 1 round lot. In equilibrium, the informed dealer never chooses a shallow book (whether $\Psi=1$ or not). Thus when she observes a shallow book, the follower does not update her beliefs and behaves as in the benchmark case. These arguments establish the second part of the proposition.

Part 2. Next we show that the informed dealer's bidding strategy is a best response. When $\Psi=1$, the argument is identical to the argument developed in the proof of the previous proposition (with $u_{T}^{*}=1$ ). When $\Psi=0$, straight forward computations yield (taking into account the follower's reaction):

$$
\Pi^{L}(T, 0)=\frac{1}{2}\left(A_{2}-v_{0}\right)=\Delta
$$

and

$$
\Pi^{L}(S, 0)=A_{1}-v_{0}=\Delta
$$

and

$$
\Pi^{L}(D, 0)=E\left(\widetilde{Q}_{u}\right)\left(A_{1}-v_{0}\right)=\frac{3}{2}\left(A_{1}-v_{0}\right)=\frac{3}{2} \Delta .
$$

Thus the informed dealer's best response when there is no information event is to post schedule $D$.

Part 3. On the equilibrium path, there is at least 1 round lot offered at price $A_{1}$. Thus the small trade spread is $\left(A_{1}-v_{0}\right)$ with certainty, as in the benchmark case. There are cases, however, in which the book will be shallow at the end of the bidding stage (instead of deep in the benchmark case). This occurs when the leader (informed or not) chooses a thin book. In this case the follower submits a limit order for 1 round lot at price $A_{1}$ and at the end of the bidding stage the book is shallow. Thus there are cases in which the marginal execution price for large market orders is $A_{2}$. This implies that the large trade spread is greater than in the benchmark case, on average.

\section{Proof of Proposition 5}

The proof is identical to the proof of Proposition 4. The only difference is that

$$
A_{1}-E_{\pi_{T}(1, \beta)}(V \mid \widetilde{Q}(2) \geq 2)=\Delta-\left(\frac{2 \pi_{T}(1, \beta)}{\pi_{T}(1, \beta) \alpha+1}\right) \alpha \sigma>0,
$$

since $\beta>\beta^{* *}$. It immediately follows that $\Pi^{F}(2, T)>\Pi^{F}(1, T)$. This means that the follower submits a limit order for 2 round lots when the book is thin. Notice that in this case, the book 
is deep with certainty at the end of the bidding stage, as in the benchmark case. This yields the last part of the proposition.

Proof of Corollary 1. It follows immediately from the arguments in the text.

\section{Proof of Corollary 2.}

In what follows, a superscript "a" (resp. "na") indexes the value of a variable in the anonymous (resp. non-anonymous) market.

Part 1. The Small Trade Spread. The expected small trade spread is given by:

$$
E S_{\text {small }}^{j}=\Delta\left(1+\operatorname{Pr}\left(Q_{1}^{j}=0\right)\right), \text { for } j \in\{a, n a\} .
$$

We deduce that the difference between the expected small trade spread in the anonymous market and the expected small trade spread in the non-anonymous markets is:

$$
E S_{\text {small }}^{a}-E S_{\text {small }}^{n a}=\Delta\left(\operatorname{Pr}\left(\widetilde{Q}_{1}^{a}=0\right)-\operatorname{Pr}\left(\widetilde{Q}_{1}^{n a}=0\right)\right) .
$$

When $\beta>\beta^{*}$, we have $\operatorname{Pr}\left(\widetilde{Q}_{1}^{a}=0\right)=0$. This follows from Propositions 4 and 5 . Furthermore we deduce from Corollary 1 that:

$$
\operatorname{Pr}\left(\widetilde{Q}_{1}^{n a}=0\right)=(1-\beta)\left(\frac{1}{8}+\frac{1-m^{*}(0)}{8}\right)>0 .
$$

Thus for $\beta>\beta^{*}, E S_{\text {small }}^{a}-E S_{\text {small }}^{\text {na }}<0$. When $\beta \leq \beta^{*}$, using the equilibrium bidding strategies described in Proposition 3, we obtain:

$$
\operatorname{Pr}\left(\widetilde{Q}_{1}^{a}=0\right)=\beta\left(\frac{\Phi_{T}}{4}\right)+(1-\beta)\left(\frac{1}{8}+\frac{1-m^{*}(\beta)}{8}\right) .
$$

Thus

$$
\operatorname{Pr}\left(\widetilde{Q}_{1}^{a}=0\right)-\operatorname{Pr}\left(\widetilde{Q}_{1}^{n a}=0\right)=\beta\left(\frac{\Phi_{T}}{4}\right)+\frac{(1-\beta)}{8}\left(m^{*}(0)-m^{*}(\beta)\right) .
$$

Using the expression for $m^{*}(\beta)$, we rewrite this equation:

$$
\operatorname{Pr}\left(\widetilde{Q}_{1}^{a}=0\right)-\operatorname{Pr}\left(\widetilde{Q}_{1}^{n a}=0\right)=\frac{\beta \Phi_{T} \alpha}{8 r}>0,
$$

which means that $E S_{\text {small }}^{a}-E S_{\text {small }}^{n a}>0$ when $\beta \leq \beta^{*}$.

Part 2. The Large Trade Spread. The expected large trade spread is given by

$$
E S_{l \arg e}^{j}=\Delta\left(2-\operatorname{Pr}\left(Q_{2}^{j}=2\right)\right), \text { for } j \in\{a, n a\} .
$$

We deduce that the difference between the expected large trade spread in the anonymous market and the expected large trade spread in the non-anonymous markets is:

$$
E S_{l \text { arg } e}^{a}-E S_{l \text { arg } e}^{n a}=\Delta\left(\operatorname{Pr}\left(\widetilde{Q}_{1}^{n a}=2\right)-\operatorname{Pr}\left(\widetilde{Q}_{1}^{a}=2\right)\right) .
$$


Using Corollary 1, we obtain

$$
\operatorname{Pr}\left(\widetilde{Q}_{1}^{n a}=2\right)=\beta+\frac{(1-\beta) m^{*}(0)}{2}<1 .
$$

When $\beta>\beta^{* *}$, we have $\operatorname{Pr}\left(\widetilde{Q}_{1}^{a}=2\right)=1$ (see Proposition 5). Thus $E S_{l \text { arg } e}^{a}-E S_{l \text { arg } e}^{n a}<0$ for $\beta>\beta^{* *}$. For $0 \leq \beta<\beta^{*}$, we deduce from Proposition 5 that:

$$
\operatorname{Pr}\left(\widetilde{Q}_{1}^{a}=2\right)=\beta\left(\Phi_{S}+\Phi_{D}\right)+\frac{(1-\beta) m^{*}(\beta)}{2} .
$$

Hence

$$
\operatorname{Pr}\left(\widetilde{Q}_{1}^{n a}=2\right)-\operatorname{Pr}\left(\widetilde{Q}_{1}^{a}=2\right)=\frac{(1-\beta)}{2}\left(m^{*}(0)-m^{*}(\beta)\right)+\beta \Phi_{T} .
$$

Using the expression for $m^{*}($.$) and rearranging, we rewrite this equation:$

$$
\operatorname{Pr}\left(\widetilde{Q}_{1}^{n a}=2\right)-\operatorname{Pr}\left(\widetilde{Q}_{1}^{a}=2\right)=\frac{\beta \Phi_{T} \alpha}{2 r}>0 .
$$

We deduce that $E S_{l \text { arg } e}^{a}-E S_{l \text { arg } e}^{n a}>0$ for $\beta<\beta^{*}$.

For $\beta^{*}<\beta \leq \beta^{* *}$, we deduce from Proposition 4 that:

$$
\operatorname{Pr}\left(\widetilde{Q}_{1}^{a}=2\right)=\beta\left(\Phi_{S}+\Phi_{D}\right)+\frac{(1-\beta)}{2} .
$$

Thus

$$
\operatorname{Pr}\left(\widetilde{Q}_{1}^{n a}=2\right)-\operatorname{Pr}\left(\widetilde{Q}_{1}^{a}=2\right)=\frac{(1-\beta)}{2}\left(m^{*}(0)-1\right)+\beta \Phi_{T}
$$

Hence,

$$
E S_{l \arg e}^{a}-E S_{l \text { arg } e}^{n a}=\Delta\left(\frac{(1-\beta)}{2}\left(m^{*}(0)-1\right)+\beta \Phi_{T}\right) .
$$

Substituting $m^{*}(0)$ by its expression, it is readily shown that the Right-Hand -Side of this expression is negative when $\beta^{*}<\beta \leq \beta^{* *}$. We deduce that $E S_{l \text { arg } e}^{a}-E S_{l \arg e}^{n a}>0$ when $\beta^{*}<\beta \leq \beta^{* *}$.

\section{Proof of Corollary 3}

Recall that :

$$
I_{B}=\sigma^{2}[\operatorname{Pr} o b(\Psi=1 \mid L a)-\operatorname{Pr} o b(\Psi=1 \mid S m)],
$$

Hence, in order to establish Corollary 3, we must compute $\operatorname{Pr} o b(\Psi=1 \mid$ Spread $=S m)$ and $\operatorname{Pr} o b(\Psi=1 \mid$ Spread $=L a)$ both in the non-anonymous and in the anonymous regime.

1) In the Non-Anonymous Regime. Using the bidding strategies described in Corollary 1 and Bayesian calculus, we obtain :

$$
\operatorname{Pr} o b^{n a}(\Psi=1 \mid \text { Spread }=S m)=\frac{\beta+(1-\beta) u_{T}^{*}}{2\left(\beta+(1-\beta) u_{T}^{*}\right)+(1-\beta)\left(1-u_{T}^{*}\right) m^{*}(0)}<\frac{1}{2},
$$

and

$$
\operatorname{Pr} o b^{n a}(\Psi=1 \mid \text { Spread }=L a)=\frac{1}{2-m^{*}(0)}>\frac{1}{2} .
$$


We conclude that $I_{B}^{n a}>0$.

2) In the Anonymous Regime. If $\beta \leq \beta^{*}$, using the bidding strategies described in Proposition 3 and Bayesian calculus, we obtain :

$\operatorname{Pr} o b^{a}(\Psi=1 \mid$ Spread $=S m)=\frac{\beta\left(\Phi_{S}+\Phi_{D}+\Phi_{T} u_{T}^{*}\right)+(1-\beta) u_{T}^{*}}{2\left(\beta\left(\Phi_{S}+\Phi_{D}+\Phi_{T} u_{T}^{*}\right)+(1-\beta) u_{T}^{*}\right)+(1-\beta)\left(1-u_{T}^{*}\right) m^{*}(\beta)}<\frac{1}{2}$, and

$$
\operatorname{Pr} o b^{a}(\Psi=1 \mid \text { Spread }=L a)=\frac{\beta \Phi_{T}+(1-\beta)}{2\left(\beta \Phi_{T}+(1-\beta)\right)-(1-\beta) m^{*}(\beta)}>\frac{1}{2} .
$$

Hence we conclude that if $\beta \leq \beta^{*}$ then $I_{B}^{a}>0$. If $\beta>\beta^{*}$, using the bidding strategies described in Propositions 4, 5 and bayesian calculus, we obtain :

$$
\operatorname{Pr} o b(\Psi=1 \mid \text { Spread }=s m)=\frac{1}{2},
$$

and

$$
\operatorname{Pr} o b^{a}(\Psi=1 \mid \text { Spread }=L a)=\frac{\beta \Phi_{T}+(1-\beta)}{2 \beta \Phi_{T}+(1-\beta)}>\frac{1}{2} .
$$

We conclude that if $\beta>\beta^{*}$ then $I_{B}^{a}>0$. In this case, it is important to realize that the large spread is necessarily established by the leader. This remark is used implicitly in the computation of $\operatorname{Pr} o b^{a}(\Psi=1 \mid$ Spread $=L a)$.

Now we compare $I_{B}^{a}$ and $I_{B}^{n a}$. First consider the case in which $\beta \leq \beta^{*}$. Using the expression of $m^{*}(\beta)$ given in Proposition 3, it is easily shown that

$$
\operatorname{Pr} o b^{a}(\Psi=1 \mid \text { Spread }=L a)=\operatorname{Pr} o b^{n a}(\Psi=1 \mid \text { Spread }=L a) .
$$

We deduce that :

$$
I_{B}^{n a}-I_{B}^{a}=\sigma^{2}\left[\operatorname{Pr} o b^{a}(\Psi=1 \mid \text { Spread }=S m)-\operatorname{Pr} o b^{n a}(\Psi=1 \mid \text { Spread }=S m)\right]
$$

Using the expressions of $\operatorname{Pr} o b^{a}(\Psi=1 \mid$ Spread $=S m)$ and $\operatorname{Pr} o b^{n a}(\Psi=1 \mid$ Spread $=S m)$, we obtain after some algebra that $I_{B}^{n a}<I_{B}^{a}$. Now consider the case in which $\beta>\beta^{*}$. In this case, it is easily shown that

$$
\operatorname{Pr} o b^{a}(\Psi=1 \mid \text { Spread }=S m)>\operatorname{Pr} o b^{n a}(\Psi=1 \mid \text { Spread }=S m),
$$

and

$$
\operatorname{Pr} o b^{a}(\Psi=1 \mid \text { Spread }=L a)<\operatorname{Pr} o b^{n a}(\Psi=1 \mid \text { Spread }=L a) .
$$

We deduce that $I_{B}^{n a}>I_{B}^{a}$ when $\beta>\beta^{*}$ 


\subsection{Appendix B}

In this appendix, we propose an extension of the baseline model in which the informed dealer faces competition from several non-informed dealer or from another informed dealer. We show that when $0 \leq \beta \leq \beta^{*}$, the equilibrium is very similar to the equilibrium described in Proposition 3 of the paper. In particular the informed dealer keeps using a bluffing strategy. For brevity, we do not present the equilibrium outcomes for the case in which $\beta>\beta^{*}$. There are qualitatively similar to those described in Propositions 4 and 5.

A.Several Uninformed Dealers. Suppose that $N \geq 1$ uninformed dealers observe the limit orders posted in the initial stage. They submit their limit orders sequentially. Now consider the following course of actions :

1. The informed dealer acts as described in Proposition 3.

2. When she faces a thin book, the uninformed dealer who reacts first submits a limit order for 1 round lot at price $A_{1}$ with probability $p_{T}^{*}(N)=1-\left(\frac{1}{4}\right)^{1 / N}$ and does nothing otherwise. For other states of the book, she acts as described in Proposition 3.

3. An uninformed dealer who does not react first submits a limit order for 1 round lot at price $A_{1}$ with probability $p_{T}^{*}(N)=1-\left(\frac{1}{4}\right)^{1 / N}$ if she faces a thin book and does nothing otherwise.

It is readily shown that these bidding strategies constitute an equilibrium (we omit the detailed proof for brevity). When they observe a thin book, uninformed dealers revise upward their beliefs about the occurence of an information event in such a way that they are all indifferent between submitting a limit order at price $A_{1}$ or not (exactly as described in Section 4.2). Hence they play a mixed strategy when they observe a thin book. Their mixed strategy is such that the probability of being undercut for the informed dealer if he posts a thin book is:

$$
u_{T}^{*}=p_{T}^{*}(N)+\left(1-p_{T}^{*}(N)\right) p_{T}^{*}(N)+\ldots+\left(1-p_{T}^{*}(N)\right)^{N-1} p_{T}^{*}(N)=3 / 4 .
$$

Thus, when there is no information event, the informed dealer is just indifferent between posting a deep book or a thin book (as explained in Section 4.2). Therefore he uses the bluffing strategy described in Proposition 3.

B.Competition between Informed Dealers. Suppose that the follower can be informed on the likelihood of an information event with probability $\theta$. Otherwise she is uninformed. Consider the following course of actions when $\theta \leq \frac{3}{4}$.

1. The informed dealer who acts in stage $L$ bids as described in Proposition 3. 
2. When she faces a thin book, the uninformed dealer submits a limit order for 1 round lot at price $A_{1}$ with probability $p_{T}^{*}=\frac{\left(\frac{3}{4}-\theta\right)}{(1-\theta)}$ and does nothing otherwise. For other states of the book, she acts as described in Proposition 3.

3. When there is an information event, the informed dealer who acts in stage $F$ does nothing. If there is no information event, the informed dealer who acts in stage $F$ submits a limit order at price $A_{1}$ for (a) 1 round lot if she faces a shallow book and (b) 2 round lots if she faces a thin book.

It is straightforward to show that these bidding strategies form an equilibrium. For brevity, we just show that it is optimal for the informed dealer acting in stage $L$ to use a bluffing strategy. When there is no information event, the probability that the follower undercuts a thin book is:

$$
u_{T}^{*}=\theta+(1-\theta) p_{T}^{*}=3 / 4 .
$$

Thus the informed dealer is just indifferent between posting a deep book or a thin book when there is no information event. Therefore he uses a bluffing strategy, as described in Proposition 3. It follows that a switch to anonymity will induce the informed dealer acting in the first stage to bid more aggressively, exactly as in the case in which $\theta=0$. Notice that this result holds for all values of $\theta \leq \frac{3}{4}$. For larger values of $\theta$, the informed dealer acting in stage $L$ does not use a bluffing strategy (he behaves as described in Propositions 4 and 5) and a switch to anonymity has no effect on market liquidity. 


\section{Figure 1}

Date 1 : Tree Diagram of the Trading Process.

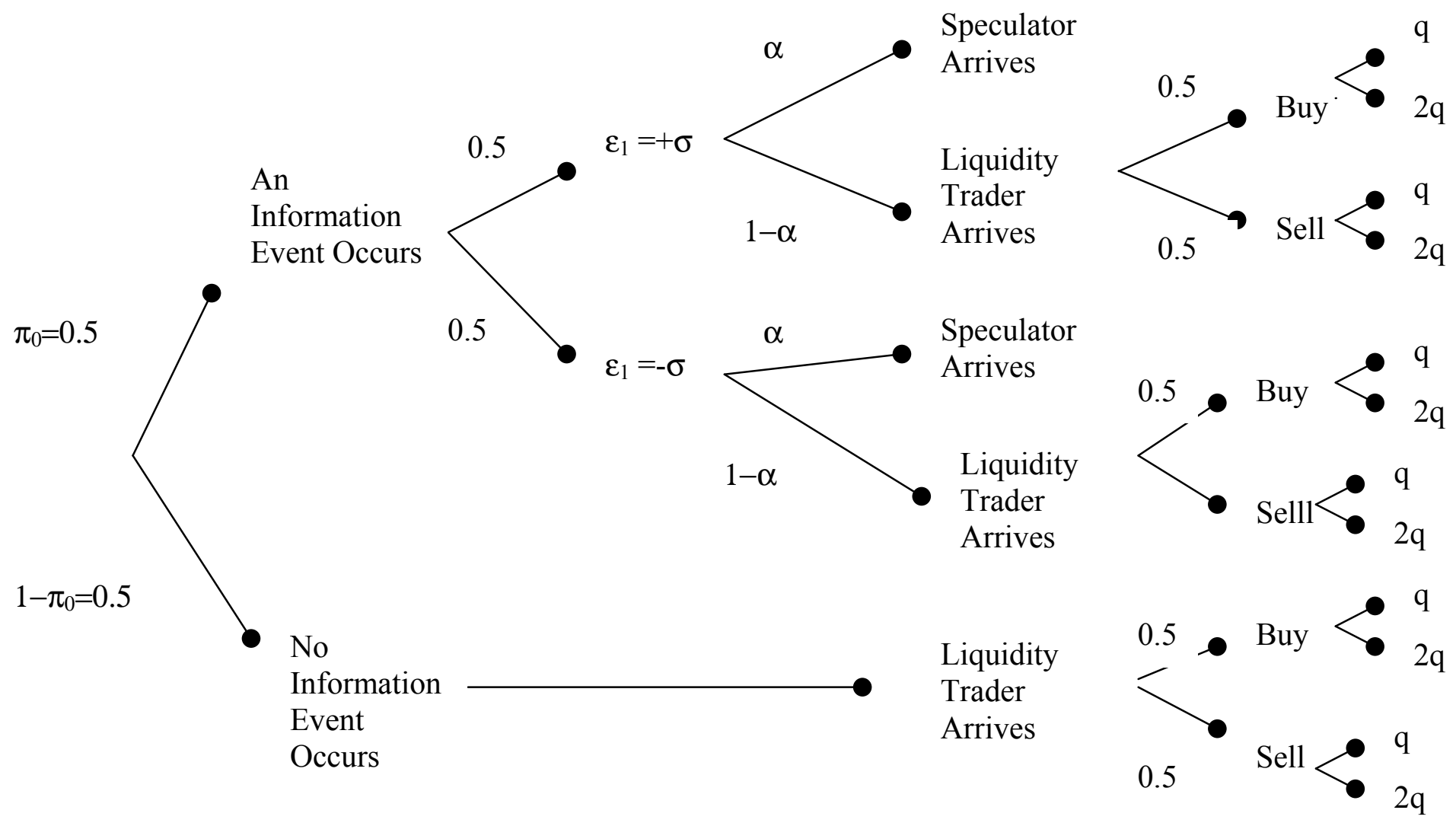

Figure 2 : Building the Limit Order Book

STAGE L

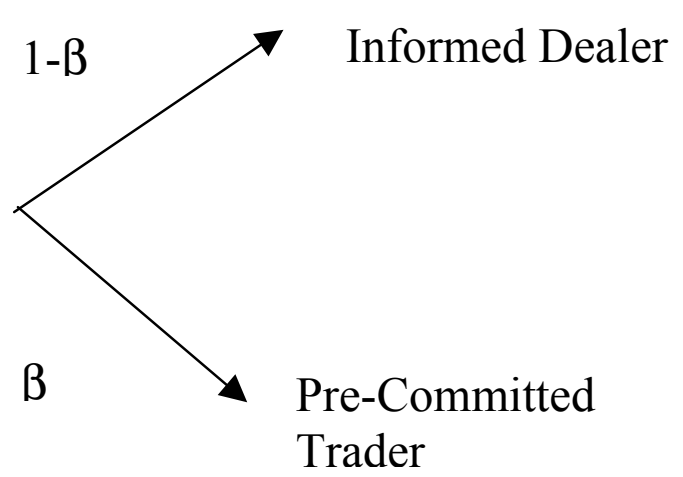

STAGE F

Uninformed Dealer

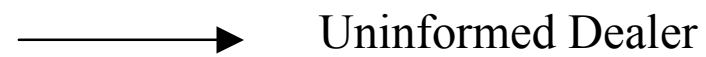


Figure 3 : Equilibrium when $\beta<\beta^{*}$

Perceived cost of submitting a limit order for 1 round lot at price $\mathrm{A}_{1}$

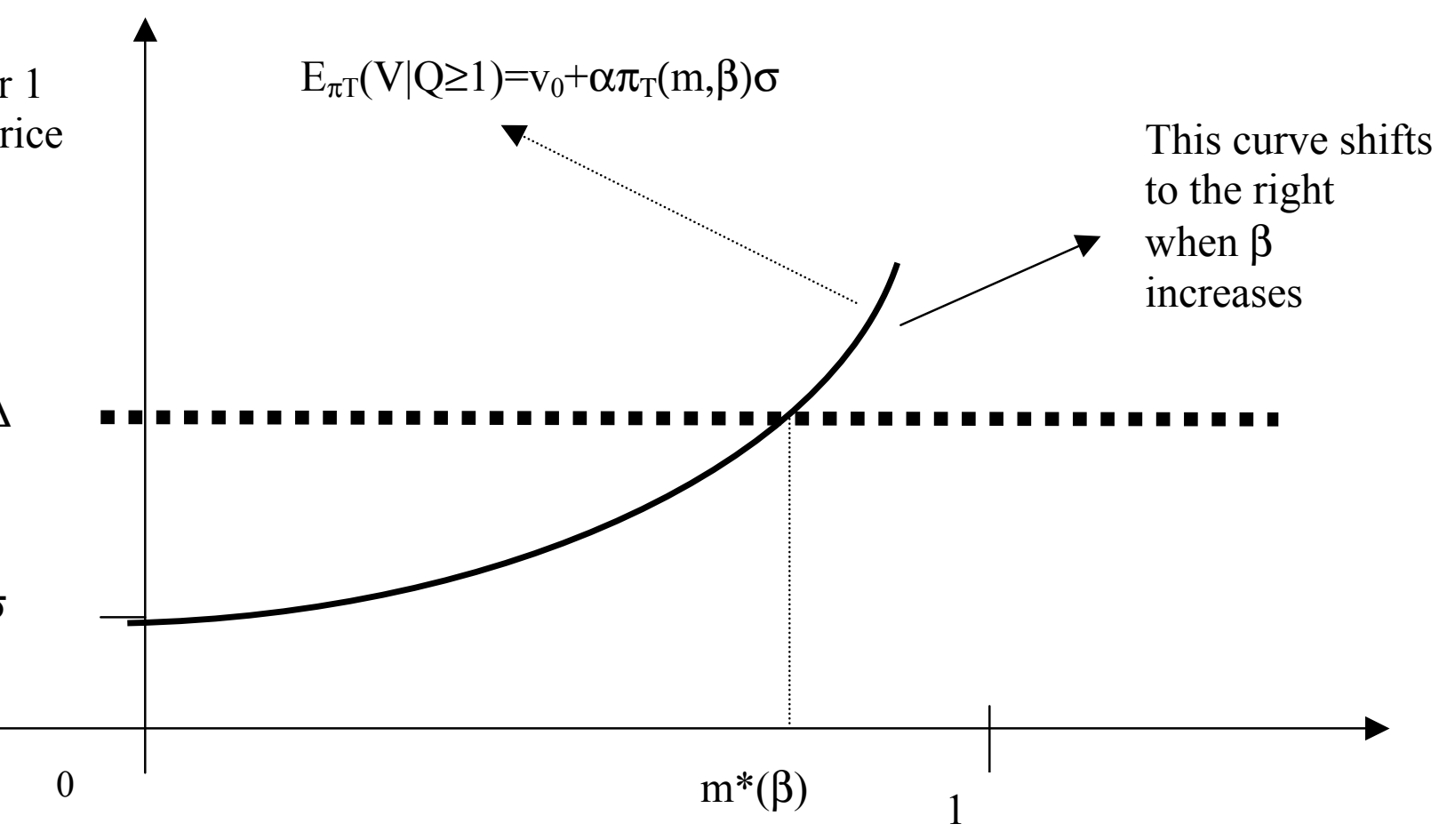

Figure 4 : Equilibrium when $\beta^{*}<\beta<\beta^{* *}$

Perceived costs of submitting a limit order for 1 or 2 round lot at price $\mathrm{A}_{1}$

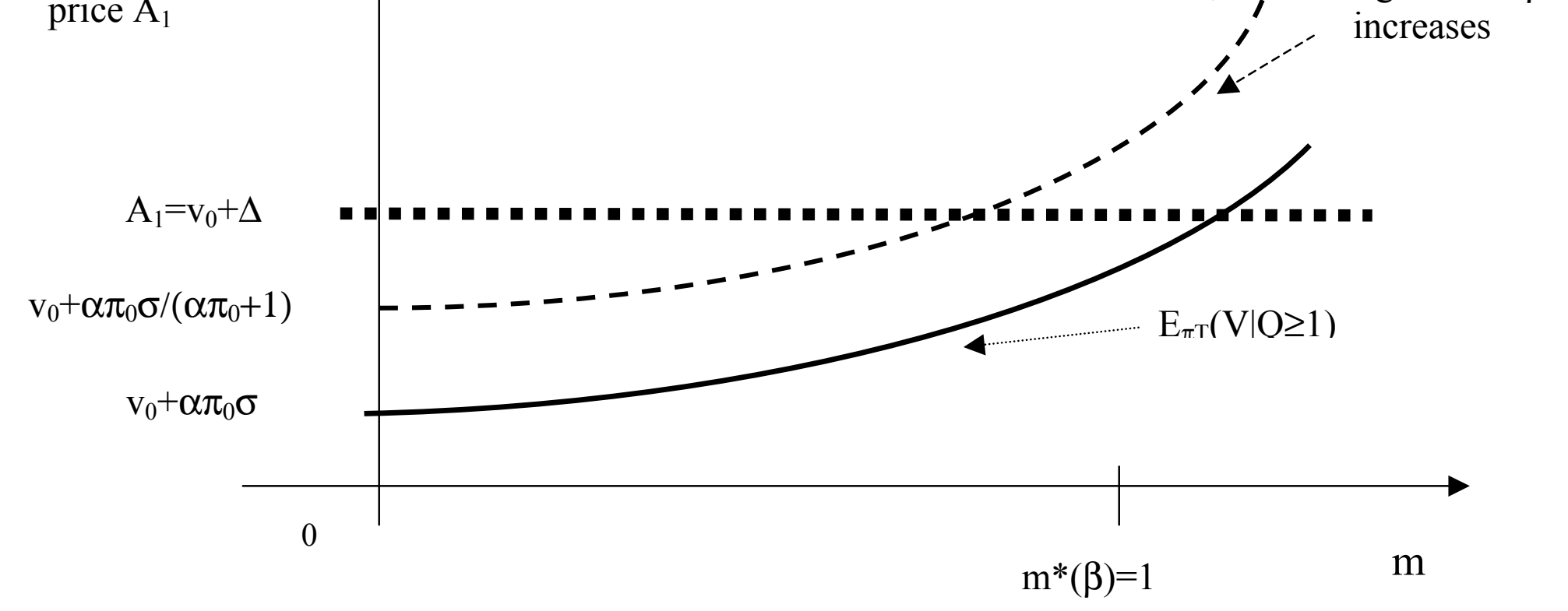


Table 3

\section{Descriptive statistics}

The table reports averages for the variables listed in the first column. We first calculated averages for each stock and each day. Then, we average over the 14 days of the pre-event period and the post-event period, respectively. Volatility is measured by the standard deviation of 30 minute midquote returns. The last two columns report the test statistics ( a t-test and $\mathrm{z}$ value for a Wilcoxon test) of the null hypothesis that the differences in means and medians, respectively, are zero.

\begin{tabular}{|c|c|c|c|c|c|}
\hline & Mean & Pre-event & Post-event & t-value & z-value \\
\hline Number of trades & 2301 & 2317 & 2286 & 0,07 & 0,33 \\
\hline Price & 87,65 & 85,43 & 89,87 & 0,33 & 0,39 \\
\hline Trading volume (shares) & 1378467 & 1323177 & 1433757 & 0,26 & 0,84 \\
\hline Trading volume ( $€$ mio) & 91 & 83 & 99 & 0,73 & 1,01 \\
\hline Average Trade Size (shares) & 488 & 460 & 515 & 0,90 & 0,85 \\
\hline Volatility & 0,0055 & 0,0063 & 0,0047 & 5,22 & 19,82 \\
\hline Market Capitalization ( $€$ mio) & 30170 & 26482 & 33857 & 0,99 & 0,40 \\
\hline
\end{tabular}


Table 4

Univariate Analysis of the Spread

The table reports averages for the variables listed in the first column. We first calculated averages for each stock and each day. Then, we average over the 14 days of the pre-event period and the post-event period, respectively. Figures in parentheses are the standard deviations of each spread measure. The last two columns report the test statistics (respectively a t-test and a Wilcoxon test) of the null hypothesis that the differences in means and medians, respectively, are zero.

\begin{tabular}{|c|c|c|c|c|c|}
\hline & Mean & Pre-event & Post-event & t-value & z-value \\
\hline $\begin{array}{r}\text { quoted percentage spread, equally-weighted (in \%) } \\
\text { (standard deviation) }\end{array}$ & $\begin{array}{c}19,56 \% \\
(0,16)\end{array}$ & $\begin{array}{c}22,04 \% \\
(0,18)\end{array}$ & $\begin{array}{c}17,09 \% \\
(0,15)\end{array}$ & 3,67 & 11,24 \\
\hline $\begin{array}{r}\text { quoted percentage spread, time-weighted (in \%) } \\
\text { (standard deviation) }\end{array}$ & $\begin{array}{c}19,50 \% \\
(0,19)\end{array}$ & $\begin{array}{c}21,91 \% \\
(0,22)\end{array}$ & $\begin{array}{c}17,09 \% \\
(0,17)\end{array}$ & 3,56 & 10,26 \\
\hline effective spread, equally-weighted & $\begin{array}{l}0,142 \\
(0,10)\end{array}$ & $\begin{array}{l}0,154 \\
(0,11)\end{array}$ & $\begin{array}{l}0,129 \\
(0,09)\end{array}$ & 1,23 & 1,11 \\
\hline
\end{tabular}


Table 5

Multivariate Analysis of the Spread

This table presents multivariate analysis of the various spread measures denoted in line 1. For all regressions we use daily data for each stock to estimate the coefficients, and we compute Newey-West standard errors with lag two to control for heteroskedasticity and autocorrelation. In Regression 1, we report the results of an OLS regression of each spread measure on the following control variables:

$\mathrm{S}_{\mathrm{i}, t}=\mathrm{a}_{0}+\mathrm{a}_{1} \log \left(\right.$ Volu $\left._{\mathrm{i}, \mathrm{t}}\right)+\mathrm{a}_{2} \mathrm{TS}_{\mathrm{i}, \mathrm{t}}+\mathrm{a}_{3} \mathrm{P}_{\mathrm{i}, \mathrm{t}}+\mathrm{a}_{4} \sigma_{\mathrm{i}, \mathrm{t}}+\mathrm{a}_{5} \mathrm{D}_{\mathrm{i}, \mathrm{t}}+\mathrm{e}_{\mathrm{i}, \mathrm{t}}$

Volume is measured in mio€. The ticksize variable measures the average effective tick size. The tick size is $1 €$-Cent ( 5 Cents, 10 Cents, 50 Cents) for stocks trading at prices below $50 €$ (between 50 and $100 €$, between 100 and $500 €$, above $500 €$ ) The effective tick size can take on intermediate values if a stock trades at prices in more than one tick size range. Volatility is measured by the standard deviation of 30 -minute midquote returns. In regression 2 , we run the same regression, but allow for stock-specific intercepts by including a dummy variable $\mathrm{D}_{\mathrm{i}}$ for each stock that is equal to one when the stock is $\mathrm{i}$ and zero otherwise.

In Regression 3, we control for cross-correlation by introducing 14 dummy variables $T_{t}$ that equal one if the day is $t(t=15, \ldots .28$ in the post-event period) and 0 otherwise. For clarity, we omit to report estimates of the intraday dummies and of the fixe effects. However, in Regression 3, we report the median of the day dummy variables. A "*" denotes significance at the $5 \%$ level.

\begin{tabular}{|c|c|c|c|c|c|c|c|c|c|c|c|c|c|c|c|}
\hline & Regression 1 & : Baseline $\mathrm{r}$ & gression & & & Regression 2 & Regression & ith fixed effe & & & Regression 3 & Regression & with fixed ef & fects and $14 \mathrm{da}$ & ay dummies \\
\hline & $\begin{array}{c}\text { quoted } \\
\text { spread in } €, \\
\text { equally- } \\
\text { weigted }\end{array}$ & $\begin{array}{c}\text { quoted } \\
\text { spread } € \text {, } \\
\text { time- } \\
\text { weighted }\end{array}$ & $\begin{array}{c}\text { quoted } \\
\text { percentage } \\
\text { spread, } \\
\text { equally- } \\
\text { weighted } \\
\text { (in } \% \text { ) }\end{array}$ & $\begin{array}{l}\text { quoted } \\
\text { percentage } \\
\text { spread, time- } \\
\text { weighted } \\
\text { (in \%) }\end{array}$ & $\begin{array}{c}\text { effective } \\
\text { spread in } € \text {, } \\
\text { equally- } \\
\text { weigted }\end{array}$ & $\begin{array}{c}\text { quoted } \\
\text { spread in } €, \\
\text { equally- } \\
\text { weigted }\end{array}$ & $\begin{array}{c}\text { quoted } \\
\text { spread } € \text {, } \\
\text { time- } \\
\text { weighted }\end{array}$ & $\begin{array}{c}\text { quoted } \\
\text { percentage } \\
\text { spread, } \\
\text { equally- } \\
\text { weighted } \\
\text { (in } \% \text { ) }\end{array}$ & $\begin{array}{c}\text { quoted } \\
\text { percentage } \\
\text { spread, time- } \\
\text { weighted } \\
\text { (in \%) }\end{array}$ & $\begin{array}{c}\text { effective } \\
\text { spread in } €, \\
\text { equally- } \\
\text { weigted }\end{array}$ & $\begin{array}{c}\text { quoted } \\
\text { spread in } €, \\
\text { equally- } \\
\text { weigted }\end{array}$ & $\begin{array}{c}\text { quoted } \\
\text { spread } €, \\
\text { time- } \\
\text { weighted }\end{array}$ & $\begin{array}{c}\text { quoted } \\
\text { percentage } \\
\text { spread, } \\
\text { equally- } \\
\text { weighted } \\
\text { (in } \% \text { ) }\end{array}$ & $\begin{array}{l}\text { quoted } \\
\text { percentage } \\
\text { spread, time- } \\
\text { weighted } \\
\text { (in \%) }\end{array}$ & $\begin{array}{c}\text { effective } \\
\text { spread in } € \\
\text { equally- } \\
\text { weigted }\end{array}$ \\
\hline Constant & $\begin{array}{c}0,101 * \\
(17,01)\end{array}$ & $\begin{array}{c}0,109 * \\
(17,49)\end{array}$ & $\begin{array}{c}0,343 * \\
(38,52)\end{array}$ & $\begin{array}{c}0,359 * \\
(40,46)\end{array}$ & $\begin{array}{c}0,055 * \\
(7,56)\end{array}$ & $\begin{array}{r}0,129 * \\
(11,02)\end{array}$ & $\begin{array}{c}0,141 * \\
(12,45)\end{array}$ & $\begin{array}{c}0,297 * \\
(22,12)\end{array}$ & $\begin{array}{c}0,315 * \\
(24,62)\end{array}$ & $\begin{array}{c}0,077 \text { * } \\
(7,60)\end{array}$ & $\begin{array}{c}0,123 * \\
(10,32)\end{array}$ & $\begin{array}{c}0,135 * \\
(11,71)\end{array}$ & $\begin{array}{c}0,296 * \\
(21,36)\end{array}$ & $\begin{array}{c}0,315 * \\
(23,49)\end{array}$ & $\begin{array}{c}0,074 * \\
(7,18)\end{array}$ \\
\hline Log(volume) & $\begin{array}{c}-0,031 * \\
(-23,43)\end{array}$ & $\begin{array}{c}-0,032 * \\
(-22,65)\end{array}$ & $\begin{array}{c}-0,047 * \\
(-28,06)\end{array}$ & $\begin{array}{c}-0,049 * \\
(-29,03)\end{array}$ & $\begin{array}{r}-0,020 * \\
(-13,88)\end{array}$ & $\begin{array}{c}-0,020 * \\
(-8,21)\end{array}$ & $\begin{array}{c}-0,023 * \\
(-8,71)\end{array}$ & $\begin{array}{c}-0,027 * \\
(-8,54)\end{array}$ & $\begin{array}{c}-0,030 * \\
(-9,16)\end{array}$ & $\begin{array}{c}-0,011 * \\
(-4,31)\end{array}$ & $\begin{array}{c}-0,018 * \\
(-6,55)\end{array}$ & $\begin{array}{c}-0,021 \text { * } \\
(-7,32)\end{array}$ & $\begin{array}{c}-0,027 * \\
(-7,54)\end{array}$ & $\begin{array}{c}-0,030 * \\
(-8,26)\end{array}$ & $\begin{array}{c}-0,009 * \\
(-3,37)\end{array}$ \\
\hline Ticksize & $\begin{array}{c}0,566 * \\
(4,67)\end{array}$ & $\begin{array}{c}0,498 * \\
(4,09)\end{array}$ & $\begin{array}{c}0,559 * \\
(5,48)\end{array}$ & $\begin{array}{c}0,551 * \\
(5,30)\end{array}$ & $\begin{array}{c}0,716 * \\
(5,31)\end{array}$ & $\begin{array}{c}1,187 * \\
(6,16)\end{array}$ & $\begin{array}{c}1,119 * \\
(5,62)\end{array}$ & $\begin{array}{c}0,825 * \\
(3,76)\end{array}$ & $\begin{array}{c}0,763 * \\
(3,52)\end{array}$ & $\begin{array}{c}1,186 * \\
(7,15)\end{array}$ & $\begin{array}{c}1,182 * \\
(6,22)\end{array}$ & $\begin{array}{c}1,116 * \\
(5,75)\end{array}$ & $\begin{array}{c}0,810 * \\
(3,71)\end{array}$ & $\begin{array}{c}0,750 * \\
(3,46)\end{array}$ & $\begin{array}{c}1,191 * \\
(7,30)\end{array}$ \\
\hline Price & $\begin{array}{c}0,0014 * \\
(15,86)\end{array}$ & $\begin{array}{c}0,0015 * \\
(16,86)\end{array}$ & $\begin{array}{c}-0,0003 * \\
(-5,20)\end{array}$ & $\begin{array}{c}-0,0003 * \\
(-4,91)\end{array}$ & $\begin{array}{c}0,0011 * \\
(14,36)\end{array}$ & $\begin{array}{r}0,0004 \\
(1,86)\end{array}$ & $\begin{array}{c}0,0005 * \\
(2,43)\end{array}$ & $\begin{array}{c}-0,0004 * \\
(-2,33)\end{array}$ & $\begin{array}{c}-0,0003 * \\
(-2,02)\end{array}$ & $\begin{array}{r}0,0003 \\
(1,65)\end{array}$ & $\begin{array}{c}0,0004 \\
(1,80)\end{array}$ & $\begin{array}{c}0,0005 * \\
(2,33)\end{array}$ & $\begin{array}{c}-0,0004 * \\
(-2,20)\end{array}$ & $\begin{array}{c}-0,0003 * \\
(-1,97)\end{array}$ & $\begin{array}{c}0,0003 \\
(1,40)\end{array}$ \\
\hline Volatility & $\begin{array}{c}7,275 * \\
(13,73)\end{array}$ & $\begin{array}{l}6,218 * \\
(11,77)\end{array}$ & $\begin{array}{l}9,689 * \\
(13,08)\end{array}$ & $\begin{array}{l}7,941 * \\
(11,04)\end{array}$ & $\begin{array}{l}6,992 * \\
(10,28)\end{array}$ & $\begin{array}{r}5,443 * \\
(10,09)\end{array}$ & $\begin{array}{c}4,698 * \\
(9,02)\end{array}$ & $\begin{array}{r}7,696 * \\
(10,46)\end{array}$ & $\begin{array}{c}6,323 * \\
(9,38)\end{array}$ & $\begin{array}{c}4,298 * \\
(7,14)\end{array}$ & $\begin{array}{c}5,495 * \\
(9,56)\end{array}$ & $\begin{array}{c}4,692 * \\
(8,47)\end{array}$ & $\begin{array}{l}7,771 * \\
(10,11)\end{array}$ & $\begin{array}{c}6,275 * \\
(8,88)\end{array}$ & $\begin{array}{c}4,259 * \\
(6,71)\end{array}$ \\
\hline $\begin{array}{l}\text { Post-Event (Median of the daily } \\
\text { dummies for Specification 3) }\end{array}$ & $\begin{array}{c}-0,024 * \\
(-7,79)\end{array}$ & $\begin{array}{c}-0,024 * \\
(-7,75)\end{array}$ & $\begin{array}{c}-0,027 * \\
(-8,53)\end{array}$ & $\begin{array}{c}-0,029 * \\
(-8,99)\end{array}$ & $\begin{array}{c}-0,017 * \\
(-5,21)\end{array}$ & $\begin{array}{c}-0,025 * \\
(-10,49)\end{array}$ & $\begin{array}{c}-0,024 * \\
(-10,28)\end{array}$ & $\begin{array}{c}-0,034 * \\
(-12,30)\end{array}$ & $\begin{array}{c}-0,034 * \\
(-12,71)\end{array}$ & $\begin{array}{c}-0,020 * \\
(-5,38)\end{array}$ & $-0,026$ * & $-0,026$ * & $-0,032 *$ & $-0,035$ * & $-0,021$ * \\
\hline Number of negative daily dummies & & & & & & & & & & & (14) & (14) & (14) & (14) & (14) \\
\hline Adj. R2 & 0,86 & 0,85 & 0,65 & 0,64 & 0,70 & 0,89 & 0,89 & 0,73 & 0,73 & 0,75 & 0,90 & 0,89 & 0,74 & 0,73 & 0,75 \\
\hline
\end{tabular}




\section{Figure 5: Effective spread}

Figure 5 reports the average daily effective spread by trade size decile (trade size is measured in euro). We first calculated the average effective spread for each stock and each day. Then, we average over the 14 days of the pre-event period and the post-event period, respectively. We also report the test statistics (a t-value and $\mathrm{z}$ value for a Wilcoxon test) of the null hypothesis that the differences in means and medians, respectively, are zero.

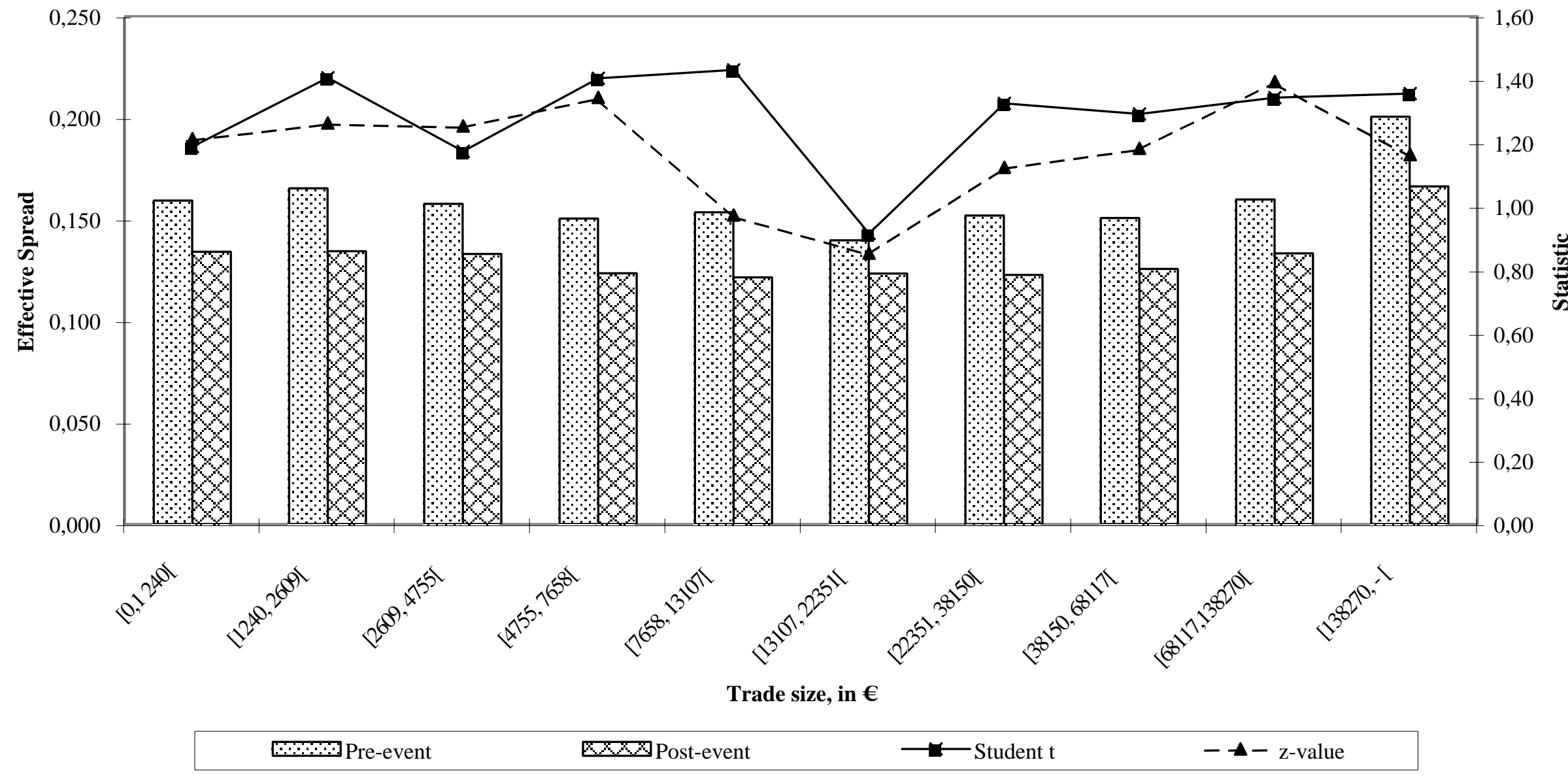




\section{Figure 6: Quoted Depth at the best quotes for various spread levels}

Figure 6 reports the average daily quoted depth at the best quotes, in $€$, in the pre and in the post-event periods. For each stock, we first compute the daily average depth for various levels of the quoted spread $(1,2, \ldots, 9$ ticks). Then, for each spread level, we average over the 14 days of the pre-event period and the post-event period, respectively. We also report the test statistics ( a t-value and a z-value for a Wilcoxon test) of the null hypothesis that the differences in means and medians between the post and the pre-event periods, respectively, are zero.

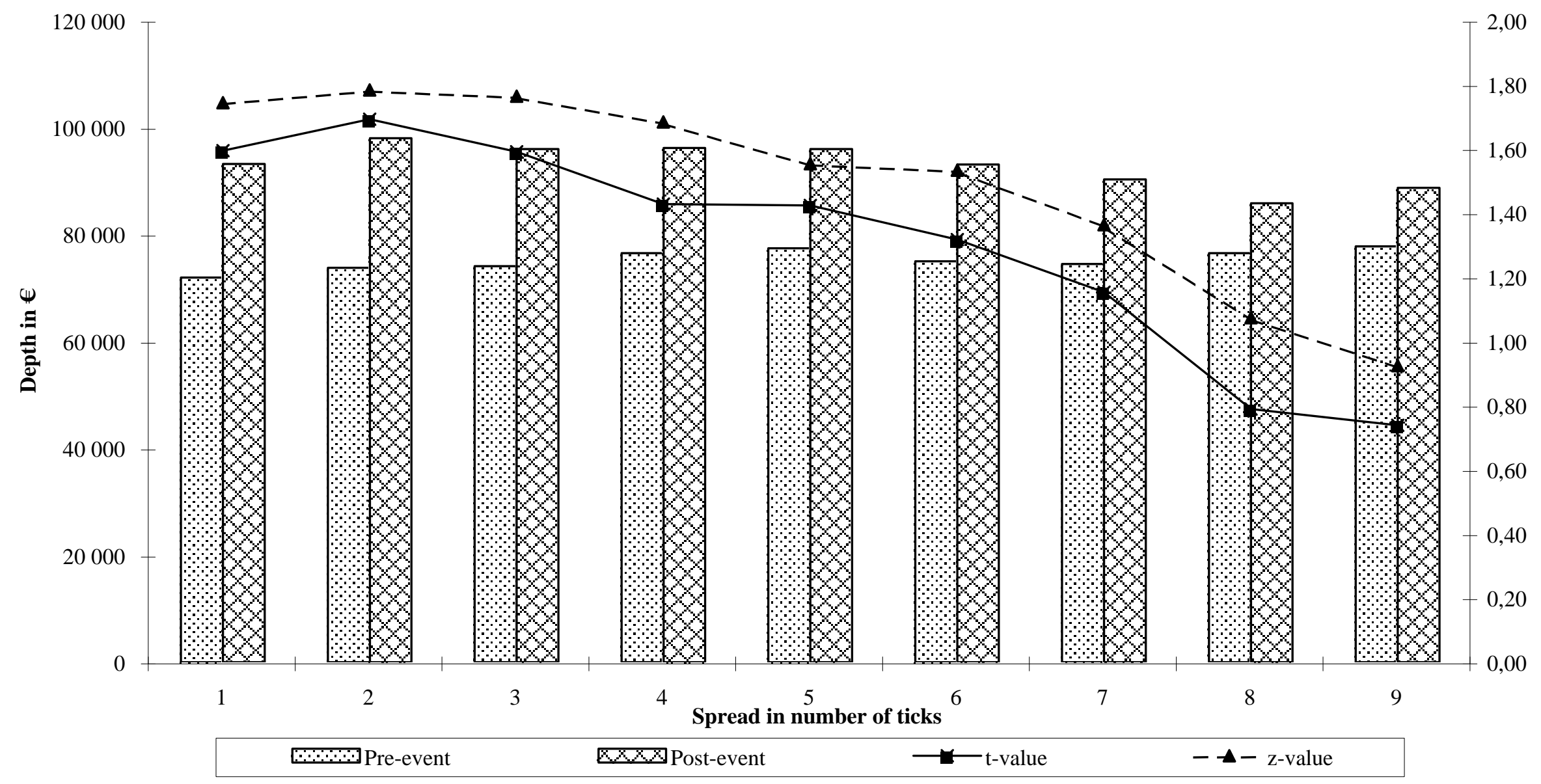


For each stock in our sample, we partition each trading day into sixteen 30-minutes intervals and one 25-minutes interval. We measure price volatility in interval $\tau \in\{1,2, \ldots, 17\}$ for stock $i$ by $\operatorname{Vol}_{i, \tau}=\left|\mathrm{m}_{\mathrm{i}, \tau}-\mathrm{m}_{\mathrm{i}, \tau-1}\right|$ where $\mathrm{m}_{\mathrm{i}, \tau}$ is the midpoint of the best buyside and sell side limit prices at the end of interval $\tau$. Then we estimate the following regression model with fixed effects:

$\mathrm{Vol}_{\mathrm{i}, \tau+1}=\mathrm{a}_{0}+\mathrm{a}_{1} \operatorname{Vol}_{\mathrm{M} \tau+1}+\mathrm{a}_{2} \operatorname{Vol}_{\mathrm{i} \tau}+\mathrm{a}_{3} \mathrm{~N}_{\mathrm{i} \tau}+\mathrm{a}_{4} \mathrm{ATr}_{\mathrm{i} \tau}+\left(\mathrm{a}_{5}+\mathrm{a}_{6} \mathrm{D}_{\mathrm{Post}}\right) \mathrm{s}_{\mathrm{i} \tau}+\sum_{\mathrm{k}=3}^{\mathrm{k}=17} \mathrm{~b}_{\mathrm{k}} \mathrm{T}_{\mathrm{k} \tau}+\sum_{\mathrm{i}=2}^{\mathrm{i}=39} \mathrm{c}_{\mathrm{i}} \mathrm{D}_{i}+\mathrm{e}_{\mathrm{i} \tau}$

where $\mathrm{s}_{\mathrm{i}, \tau}$ is the average quoted spread in interval $\tau, \mathrm{D}_{\text {post }}$ is a dummy variable equal to 1 in the post event-period and zero in the pre-event period, $\mathrm{N}_{\tau}$ is the number of transactions in interval $\tau, \operatorname{ATr}_{\tau}$ is the average trade size in interval $\tau, \mathrm{D}_{\mathrm{i}}$ is a dummy variable equal to one when the stock is $\mathrm{i}$ and zero otherwise, $\mathrm{Tk}, \tau$ is a dummy variable equal to 1 if $\mathrm{k}=\tau$ and $\mathrm{Vol}_{\mathrm{M} \tau+1}$ is a proxy for the market volatility in interval $\tau$ defined as:

$\operatorname{Vol}_{M \tau}=\frac{1}{39}\left|\sum_{i=1}^{39}\left(m_{i \tau}-m_{i \tau-1}\right)\right|$

We have partitioned each trading day into seventeen intervals but we only have sixteen intraday observations per stock since we use lagged variables as independent variables. Furthermore we drop one dummy variable for the time intervals to avoid linear dependance. The second column reports t-values for each coefficient.

A $" * "$ denotes significance at the $5 \%$ level. For clarity, we omit to report estimates of the intraday dummies and of the fixed effects.

\begin{tabular}{|c|c|c|}
\hline \multirow[b]{2}{*}{ Volatility in $[\mathrm{t}, \mathrm{t}+1]$} & \multicolumn{2}{|c|}{ Overall } \\
\hline & Coefficient & t-value \\
\hline Constant & $0,06 *$ & $(3,13)$ \\
\hline Volatility in $[\mathrm{t}-1, \mathrm{t}]$ & $0,10 *$ & $(7,12)$ \\
\hline Average spread in $[\mathrm{t}-1, \mathrm{t}]$ & $0,29 *$ & $(5,42)$ \\
\hline Average spread in $[\mathrm{t}-1, \mathrm{t}] *$ Dummy Post & $-0,23 *$ & $(5,39)$ \\
\hline Number of trades in 1,000 in $[t, t+1]$ & $0,060 *$ & $(2,75)$ \\
\hline Average transaction size in 1,000 shares in $[\mathrm{t}, \mathrm{t}+1]$ & 0,006 & $(1,28)$ \\
\hline Market volatility & $0,57 *$ & $(18,91)$ \\
\hline $\mathrm{R}^{2}$ & & \\
\hline
\end{tabular}


Table 7

For each day and each stock, we compute the number of block trades taking place in the upstairs market and in the downstairs market. A block trade is defined as a trade larger than one "Normal Block Size". Then we average across days and across stocks. We compute the average daily volume of block trades in the same way. The last column reports the test statistics of the null hypothesis that the differences in means is zero. A "*" denotes significance at the $5 \%$ level.*

\begin{tabular}{|c|c|c|c|c|c|}
\hline & Mean & Pre-event & Post-event & $\begin{array}{c}\text { Diff. } \\
\text { Post-Pre }\end{array}$ & t-value \\
\hline Downstairs Trades & & & & & \\
\hline Daily Number of block trades & 1.6 & 1.5 & 1.7 & 0.2 & 0.20 \\
\hline $\begin{array}{c}\text { Daily Volume of block trades } \\
\text { (in 1,000 €) }\end{array}$ & 101596 & 71057 & 132135 & 61078 & 1.59 \\
\hline Upstairs Trades & & & & & \\
\hline $\begin{array}{c}\text { Daily Number of block trades } \\
\text { Daily Volume of block trades } \\
\text { (in 1,000 } \mathbf{~ ) ~}\end{array}$ & 3.1 & 3.7 & 2.4 & -1.3 & $1.9827^{*}$ \\
\hline
\end{tabular}

